\title{
THE COHOMOLOGY OF PRESHEAVES OF ALGEBRAS. I: PRESHEAVES OVER A PARTIALLY ORDERED SET
}

\author{
MURRAY GERSTENHABER AND SAMUEL D. SCHACK
}

\begin{abstract}
To each presheaf (over a poset) of associative algebras $\mathbb{A}$ we associate an algebra $\mathbb{A}$ !. We define a full exact embedding of the category of (presheaf) $\mathbb{A}$-bimodules in that of $\mathbb{A}$ !-bimodules. We show that this embedding preserves neither enough (relative) injectives nor enough (relative) projectives, but nonetheless preserves (relative) Yoneda cohomology. The cohomology isomorphism links the deformations of manifolds, algebraic presheaves, and algebras. It also implies that the cohomology of any triangulable space is isomorphic to the Hochschild cohomology of an associative algebra. (The latter isomorphism preserves all known cohomology operations.) We conclude the paper by exhibiting for each associative algebra and triangulable space a "product" which is again an associative algebra.
\end{abstract}

0. Introduction. Presheaves are rather familiar objects and arise in many situations, some of which are discussed below. Our primary purpose in this paper is to prove a theorem which reduces questions about the cohomology of presheaves of rings to questions about the (classical) cohomology of rings. Specifically, fix a commutative ring $k$ and a partially ordered set (poset) $\mathscr{I}$. Let $\mathbb{A}$ be a presheaf of associative $k$-algebras over $\mathscr{I}$ hereinafter: a diagram. Then $\mathbb{A}$ has naturally associated with it an abelian category of bimodules and a (relative) Yoneda Cohomology bifunctor $\operatorname{Ext}_{\mathbf{A}-\mathbf{A}}^{\bullet}(-,-)$. (All the foregoing formalism is introduced in $\S 1$.) We define for each such diagram $\mathbb{A}$ a single $k$-algebra $\mathbb{A}$ ! and a full exact embedding, $\mathbb{M} \rightsquigarrow \mathbb{M}$ !, of the category of $\mathbb{A}$-bimodules in the category of $\mathbb{A}$ !-bimodules (§2). The embedding induces a natural transformation of Yoneda cohomology bifunctors, $\omega^{*}: \operatorname{Ext}_{\mathrm{A}-\mathrm{A}}^{\bullet}(-,-) \rightarrow \operatorname{Ext}_{\mathrm{A} !-\mathrm{A} !}^{\bullet}(-!,-!)(\S 2)$. Our central result is the Special Cohomology Comparison Theorem (CCT), namely:

for an arbitrary diagram $\mathbb{A}$ over an arbitrary poset $\omega^{\bullet}: \operatorname{Ext}_{\mathbf{A}-\mathbf{A}}^{\bullet}(-,-) \rightarrow \operatorname{Ext}_{\mathbf{A} !-\mathrm{A} !}^{\bullet}(-!,-!)$ is an isomorphism.

The proof occupies $\S \S 3,4$. The scheme of the proof will be given shortly. First we describe some of the history and utility of the CCT.

In [GS1] we proved a "primitive" version of the CCT, applicable to arbitrary diagrams defined over arbitrary finite posets and to a narrow class of diagrams defined over a limited class of infinite posets. The proofs given there for the finite

Received by the editors March 6, 1987.

1980 Mathematics Subject Classification (1985 Revision). Primary 16A58, 16A61, 16A62, 18G10, 18G25; Secondary 18E25, 32G05, 55N25, 55U10.

Key words and phrases. Associative algebra, bimodule, Yoneda cohomology, Hochschild cohomology, simplicial cohomology, deformation, triangulable space.

Both authors gratefully acknowledge the support of the National Science Foundation during the preparation of this manuscript. 
case and the few infinite cases were wholly different. Here we remove all restrictions on the diagrams and the posets and give a single proof, applicable to all cases and bearing no resemblance to either of the proofs in [GS1].

The work of [GS1] was initiated-as its title insists - to study the deformations of a diagram. (These are defined in a way naturally extending the definition for the classical case of a single algebra (cf. [G2, GS1].) Indeed, there is a map, $\operatorname{Def}(\mathbb{A}) \rightarrow \operatorname{Def}(\mathbb{A} !)$, from the set of deformations of $\mathbb{A}$ to the set of deformations of $\mathbb{A}$ !. In [GS1, §21] we analyze this map. We show there that it is an injection if, for example, $\mathbb{A}$ factors through the category of commutative algebras and $\omega^{\bullet}: \operatorname{Ext}_{\mathbb{A}-\mathbb{A}}^{\bullet}(-,-) \rightarrow \operatorname{Ext}_{\mathbb{A} !-\mathbb{A} !}^{\bullet}(-!,-!)$ is an isomorphism. The latter requirement may now be dropped from the hypotheses since it is a particular case of the CCT. In contrast, we construct from any diagram $\mathbb{A}$ another diagram ${ }^{\#} \mathbb{A}$ and a bijection $\operatorname{Def}(\mathbb{A}) \rightarrow \operatorname{Def}\left({ }^{\#} \mathbb{A}\right)$; then, using the $C C T$, we show: when the underlying poset is finite, $\operatorname{Def}(\mathbb{A}) \rightarrow \operatorname{Def}((\# \mathbb{A})$ !) is onto [GS1, §21]. Thus every diagram $\mathbb{A}$ (over a finite poset $\mathscr{I})$ has an associated ring whose deformations are "snapshots" of the deformations of the diagram, namely $(\# \mathbb{A})$ !. If, further, the algebra $\mathbb{A}(i)$ is commutative for every $i \in \mathscr{I}$ then $\operatorname{Def}(\mathbb{A}) \rightarrow \operatorname{Def}((\# \mathbb{A}) !)$ is a bijection and, so, the deformations of such a diagram are mirrored by those of an algebra. This correspondence provides a bridge between deformations of manifolds and deformations of algebras. Specifically, if $\mathscr{X}$ is a complex manifold and $\mathscr{I}$ is the poset of coordinate neighborhoods of $\mathscr{X}$ then $\mathscr{X}$ is completely determined by the presheaf $\mathbb{A}$ of holomorphic functions on $\mathscr{X}$, which is a diagram of commutative algebras. A formal deformation of $\mathscr{X}$ induces a deformation of $\mathbb{A}$. The converse is true for "commutative" deformations of $\mathbb{A}$. When $\mathscr{X}$ is compact we may even restrict $\mathscr{I}$ to be a finite Stein covering of $\mathscr{X}$ closed under intersection. Once again $\mathbb{A}$ completely determines $\mathscr{X}$ and now $\operatorname{Def}(\mathbb{A}) \rightarrow \operatorname{Def}(\mathbb{A} !)$ is a bijection. The ultimate goal of such a correspondence, clearly, is to transfer back to manifolds (or schemes or varieties) information obtained by purely algebraic means.

The value of the CCT transcends its applications to deformation theory. For example, let $\Sigma$ be a simplicial complex and let $\mathscr{I}$ be the poset formed by its simplices (ordered by incidence). If $\mathbb{k} \mathscr{F}=\mathbb{k}$ is the constant diagram (over $\mathscr{f}$ ) then there is a natural isomorphism $H^{\bullet}(\Sigma, k) \cong H^{\bullet}(\mathbb{k}$ !, $\mathbb{k}$ !) between the simplicial cohomology of $\Sigma$ and the Hochschild cohomology of $\mathbb{k}$ ! (mnemonic: $\mathrm{SC}=\mathrm{HC}$ ). This is a consequence of the classical isomorphism $H^{\bullet}(\mathbb{k} !,-) \cong \operatorname{Ext}_{\mathbf{k} !-k !}^{\bullet}(\mathbb{k} !,-)$, the (relatively easy) observation that $\operatorname{Ext}_{\mathbf{k}-\mathbf{k}}^{\bullet}(\mathbb{k}, \mathbb{k})=H^{\bullet}\left(\Sigma^{\prime}, k\right)$ where $\Sigma^{\prime}$ is the barycentric subdivision of $\Sigma$, and the CCT. It is significant that $\mathrm{SC}=\mathrm{HC}$ compares simplicial and Hochschild cohomologies. For these two cohomologies share the uncommon feature that they have "additional" cohomology operations beyond the usual cup product. [S, G1, GS2]. The explicit description of the isomorphism elucidates the similarities between the two cohomology theories and shows exactly where and why they diverge [GS2]. The few infinite cases of the CCT proven in [GS1] were strong enough to yield $\mathrm{SC}=\mathrm{HC}$ for locally finite simplicial complexes [GS2]. With the full CCT in tow SC=HC now follows for arbitrary simplicial complexes $(\S 7)$. (For finite simplicial complexes one can bypass the CCT in proving $\mathrm{SC}=\mathrm{HC}$. This is done in [GS4] and sketched in $\S 7$ of this paper.) The (classical) local cohomology of simplicial complexes is also captured by Hochschild cohomology ( $\S 7)$. 
The CCT is a potentially fruitful source for examples of algebras with prescribed cohomological properties. Indeed, in [GS4] we use $\mathrm{SC}=\mathrm{HC}$ to construct examples of analytically rigid algebras which are not infinitesimally rigid by finding spaces with the "right" cohomological properties. Each example is then an appropriate $\mathbf{k}_{\mathscr{f}}$ !. We believe that the potential for such applications is vast. To illustrate this we show in $\S 7$ that if $A$ is an associative algebra and $\mathscr{X}$ is a triangulable space then there is an algebra which behaves cohomologically as though it were the product of $A$ and $\mathscr{X}$. The algebra is just $\mathbb{A}$ ! for a diagram constructed from $A$ and $\mathscr{X}$. The algebra $\mathbb{A}$ ! would be present in any case; it is the CCT which suggests our interpretation.

Given the utility we ascribe to the CCT it seems reasonable to ask whether it is true for diagrams over an arbitrary small category, not merely a poset. The answer is no. However, there is a remedy. Both the difficulty and the remedy are discussed in the concluding remarks to $\S 2$ and will appear in [GS5, 6].

If $A$ is an associative algebra then the Hochschild cohomology $H^{\bullet}(A,-)$ is equal to $\operatorname{Ext}_{A-A}^{\bullet}(A,-)$ and can be computed as the homology of a cochain complex $C^{\bullet}(A,-)$. Similarly, for a diagram $\mathbb{A}$ there is a cochain complex $C^{\bullet}(\mathbb{A},-)$ whose homology $H^{\bullet}(\mathbb{A},-)$ is equal to $\operatorname{Ext}_{\mathbb{A}-\mathbb{A}}^{\bullet}(\mathbb{A},-)$. The definition of $C^{\bullet}(\mathbb{A},-)$ and a proof that $H^{\bullet}(\mathbb{A},-)=H\left(C^{\bullet}(\mathbb{A},-)\right)=\operatorname{Ext}_{\mathbb{A}-\mathbf{A}}^{\bullet}(\mathbb{A},-)$ were given in $[$ GS1]. We repeat the definition, but not the proof, in $\S 6$. Since, according to the CCT, $H^{\bullet}(\mathbb{A},-) \cong$ $H^{\bullet}\left(\mathbb{A}\right.$ !, - !) it is natural to ask for a cochain map $\tau^{\bullet}: C^{\bullet}(\mathbb{A},-) \rightarrow C^{\bullet}(\mathbb{A} !,-$ !) which induces the isomorphism. We introduced $\tau^{\bullet}$ in [GS1] and showed that whenever the map $\omega^{\bullet}$ is an isomorphism it follows that $H\left(\tau^{\bullet}\right)=\omega^{\bullet}$. Consequently, we can now assert: $H\left(\tau^{\bullet}\right)$ is an isomorphism for any diagram $\mathbb{A}$ over any poset $\mathscr{J}$. The cochain map $\tau^{\bullet}$ plays a critical role in both the analysis and the correspondence $\operatorname{Def}(\mathbb{A}) \rightarrow \operatorname{Def}(\mathbb{A} !),[$ GS1, $§ 21]$, and that of the relationship between simplicial and Hochschild cohomology operations, [GS2, §6]. We repeat the definition of $\tau^{\bullet}$ in $\S 6$ where we also show directly, without invoking the CCT, that $H\left(\tau^{\bullet}\right)$ is an isomorphism whenever $\mathbb{A}$ is a diagram over a finite poset. (We were unable to do this in [GS1].) We thus have a pedestrian proof of a limited, but nonetheless significant, portion of the CCT, namely: $\operatorname{Ext}_{\mathrm{A}-\mathrm{A}}^{\bullet}(-,-) \rightarrow \operatorname{Ext}_{\mathrm{A} !-\mathrm{A} !}^{\bullet}(-!,-!)$ is an isomorphism for any diagram $\mathbb{A}$ over a finite poset $\mathscr{I}$. It should be noted that this proof, where it applies, is less conceptual than that contained in $\S \S 3,4$. In particular the provenance of $\tau^{\bullet}$ remains a mystery.

Since the Hochschild cohomology of an algebra has several cohomology operations the CCT implies that $H^{\bullet}(\mathbb{A}, \mathbb{A})$ must have such operations as well. (These include a graded commutative cup product, a graded Lie bracket, and a Steenrod square.) Using $\tau^{\bullet}$ the structure of $H^{\bullet}\left(\mathbb{A}\right.$ !, $\mathbb{A}$ !) can be transported back to $H^{\bullet}(\mathbb{A}, \mathbb{A})$ and then redescribed intrinsically (i.e. without reference to $\mathbb{A} !$ and $\tau^{\bullet}$ ). We did this in [GS2, §4] but the formulae did not reach the printed page intact. Consequently, we repeat them in $\S 6$. With the formulae in hand we proved, [GS2, §5], that they had the correct properties for arbitrary diagrams over a wide class of posets, including many cases for which we did not yet know the CCT. Given the full CCT, it is now a triviality that the formulae are always "correct".

As to the proof of the CCT, first note that it would be trivial if $\mathbb{M} \leadsto \mathbb{M}$ ! were to preserve either enough projectives or enough injectives. We show in $\S 5$ that such is not the case. The proof itself begins with two reductions, the first of which 
is elementary. We show that the CCT is equivalent to the Acyclicity Theorem: if $\mathbb{E}$ is an injective $\mathbb{A}$-bimodule and $\mathbb{N}$ is an arbitrary $\mathbb{A}$-bimodule then $\mathbb{E}$ ! is a $\operatorname{Hom}_{\mathbb{A} !-\mathbb{A} !}(\mathbb{N}$ !, - )-acyclic bimodule $(\S 2)$. The second reduction is the equivalence of the CCT to the Second Acyclicity Theorem. This states that we need prove the Acyclicity Theorem only when the poset $\mathscr{I}$ is a cone-i.e. $\exists 0 \in \mathscr{I}$ with $i \geq 0$ for all $i \in \mathscr{I}$-and then only for a particular type of injective which we term 0 -primitive in $\S 1$. To finish we engage in a delicate analysis of extensions $(\S 4)$. That analysis is modelled on-but is considerably less clumsy than-the proof given in [GS3, §3] for diagrams over the poset $\mathscr{I}=\{0<1\}$. (On the other hand, there are difficulties in the general case which are invisible in the case $\mathscr{I}=\{0<1\}$. See the remarks following Theorem 3.8 and those concluding $\S 4$.)

We adhere to the following notational conventions: $k$ will be a commutative associative ring with unit. All $k$-algebras will be associative and will have a unit; all $k$-algebra maps will be unital. The category of such algebras and maps will be denoted $k$-alg. When $A$ and $B$ are $k$-algebras, a left $A$, right $B$ module will be reffered to as an $(A-B)$-bimodule. The category of such bimodules will be denoted ( $A$-B)-bimod; when $A=B$ we abbreviate this to $A$-bimod. We also abbreviate $\operatorname{Hom}_{A-b i m o d}(N, M)$ to $\operatorname{Hom}_{A-A}(N, M)$ and adopt other similar such abbreviations without comment whenever convenient. All bimodules will be assumed to be unital and to be $k$-symmetric, ( $a m=m a$ for all $a \in k$ and all $m$ in the bimodule). Since a left $A$-module is, implicitly, a right $k$-module we designate the category of left $A$ modules by $(A-k)$-bimod. Similarly, $(k-A)$-bimod is the category of right $A$-modules. We shall use + and $\otimes$ for direct sum and tensor product in $k$-bimod; otherwise we use $\oplus$ and $\otimes_{A}$. Likewise $\Pi$ and $\amalg$, when used without comment, indicate product and coproduct in $k$-bimod only. Finally, we use $\rightarrow$ and $\rightarrow$ to represent, respectively, monomorphisms and epimorphisms.

1. Rudimentary theory of diagrams. We view a poset $\mathscr{I}$ as a category in the usual way: corresponding to each order relation $i \leq j$ there is a unique map $i j: i \rightarrow j$. (In particular, $i i=\mathrm{Id}_{i}$.) A presheaf of $k$-algebras over $\mathscr{J}$ is a functor $\mathbb{A}: \mathscr{J}^{\text {op }} \rightarrow k$-alg. For brevity, we refer to $\mathbb{A}$ as a diagram (over $\mathscr{I}$ ), write $\mathbb{A}^{i}$ for $\mathbb{A}(i)$, and write $\varphi^{i j}: \mathbb{A}^{j} \rightarrow \mathbb{A}^{i}$ for $\mathbb{A}(i j)$. In particular, $\varphi^{i i}$ is the identity map of $\mathbb{A}^{i}$. The constant diagram $\mathbb{k}$ is defined by $\mathbb{k}^{i}=\mathbb{k}$ for all $i \in \mathscr{I}$ and $\varphi^{i j}=\operatorname{Id}_{k}$ for all $i \leq j$. Note that $\mathbb{k}$ is the coterminator in the category of diagrams over $\mathscr{J}$.

An $\mathbb{A}$-bimodule $\mathbb{M}$ is a presheaf of abelian groups in which (with similar notational conventions):

(1) $\mathbb{M}^{i}$ is an $\mathbb{A}^{i}$-bimodule;

(2) If $i \leq j$ then $T^{i j}: \mathbb{M}^{j} \rightarrow \mathbb{M}^{i}$ is an $\mathbb{A}^{j}$-bimodule map. (N.B. The map $\varphi^{i j}: \mathbb{A}^{j} \rightarrow \mathbb{A}^{i}$ induces a forgetful functor $\mathbb{A}^{i}$-bimod $\rightarrow \mathbb{A}^{j}$-bimod by means of which we view $\mathbb{M}^{i}$ as an $\mathbb{A}^{j}$-bimodule.) As before, $T^{i i}$ is an identity map. On occasion, particularly when considering more than one bimodule, we may revert to standard functorial notation and write $\mathbb{M}(i j)$ rather than $T^{i j}$. An $\mathbb{A}$-bimodule map $\eta: \mathbb{N} \rightarrow \mathbb{M}$ is simply a natural transformation in which each $\eta^{i}: \mathbb{N}^{i} \rightarrow \mathbb{M}^{i}$ is an $\mathbb{A}^{i}$-bimodule map. The category of $\mathbb{A}$-bimodules will denoted $\mathbb{A}$-bimod. It is abelian, complete, and cocomplete. (All constructions are made "objectwise".)

Clearly, when $\mathscr{I}$ consists of a single element these concepts reduce to the classical definitions of a $k$-algebra $A$, an $A$-bimodule $M$, and an $A$-bimodule map $f: N \rightarrow M$. We shall refer to this situation as the classical case. Traditionally, certain allowable 
maps play a critical role in the classical case. An $A$-bimodule map $f: N \rightarrow M$ is allowable if there is a $k$-bimodule map $\kappa: M \rightarrow N$ for which $f \kappa f=f$. (An epi or mono is then allowable $\Leftrightarrow$ it splits in $k$-bimod; a map is allowable $\Leftrightarrow$ in every epi-mono factorization both maps are allowable; when $k$ is a field all maps are allowable.) Concepts such as projectivity and injectivity are then defined "relative to allowable maps." These practices have risen to enable certain familiar and useful homological properties to generalize well from the case in which $k$ is a field to that in which $k$ is an arbitrary commutative ring.

So, too, for an arbitrary diagram $\mathbb{A}$ we shall consider allowable $\mathbb{A}$-bimodule maps. A map $\eta: \mathbb{N} \rightarrow \mathbb{M}$ is allowable if and only if each $\eta^{i}: \mathbb{N}^{i} \rightarrow \mathbb{M}^{i}$ is allowable. (N.B. We do not insist that the $k$-bimodule splitting maps $\kappa^{i}: \mathbb{M}^{i} \rightarrow \mathbb{N}^{i}$ comprise a $\mathbf{k}$-bimodule map $\mathbb{M} \rightarrow \mathbb{N}$.) An $\mathbb{A}$-bimodule $\mathbb{E}$ is a relative injective if for every allowable monomorphism $\mathbb{N} \rightarrow \mathbb{M}$ the induced map $\operatorname{Hom}_{\mathbf{A}-\mathbf{A}}(\mathbb{M}, \mathbb{E}) \rightarrow \operatorname{Hom}_{\mathbf{A}-\mathbf{A}}(\mathbb{N}, \mathbb{E})$ is an epimorphism. A relative injective allowable resolution of an $\mathbb{A}$-bimodule $\mathbb{M}$ is an exact sequence $0 \rightarrow \mathbb{M} \rightarrow \mathbb{E}_{0} \rightarrow \mathbb{E}_{1} \rightarrow \cdots \rightarrow \mathbb{E}_{n} \rightarrow \cdots$ in which all maps are allowable and each $\mathbb{E}_{n}$ is a relative injective $\mathbb{A}$-bimodule. Every $\mathbb{A}$-bimodule $\mathbb{M}$ has such a resolution, i.e.

THEOREM 1.1. A-bimod has enough relative injectives.

Before proving this we shall collect some elementary observations in a lemma.

LEMMA 1.2. (1) For each $i \in \mathscr{I}$ the restriction functor $\langle i\rangle^{*}: \mathbb{A}$-bimod $\rightarrow$ $\mathbb{A}^{i}$-bimod defined by $\langle i\rangle^{*} \mathbb{M}=\mathbb{M}^{i}$ is exact and preserves allowability.

(2) $\langle i\rangle^{*}$ has a right inverse $\langle i\rangle_{*}: \mathbb{A}^{i}$-bimod $\rightarrow \mathbb{A}$-bimod defined as follows: $\left(\langle i\rangle_{*} M\right)^{j}$ $=M$ if $j \geq i$ and $\left(\langle i\rangle_{*} M\right)^{j}=0$ otherwise; $\left(\langle i\rangle_{*} M\right)^{j} \rightarrow\left(\langle i\rangle_{*} M\right)^{h}$ is $\operatorname{Id}_{M}$ if $j \geq h \geq i$ and is 0 otherwise.

(3) $\langle i\rangle_{*}$ is right adjoint to $\langle i\rangle^{*}$.

(4) $\langle i\rangle_{*}$ is exact and preserves both allowability and relative injectivity.

(5) $\langle i\rangle_{*} E$ is a relative injective $\mathbb{A}$-bimodule if and only if $E$ is a relative injective $\mathbb{A}^{i}$-bimodule.

Proof. To begin, (1) and (2) are clear. The first two claims of (4) are also trivial and the third is established by the usual proof that a right adjoint to an exact functor preserves injectivity. Half of (5) is subsumed by (4). For the other half let $g \in \operatorname{Hom}_{\mathrm{A}^{i}-\mathrm{A}^{i}}(N, M)$ be an allowable monomorphism and suppose that $M \rightarrow E$ is an arbitrary $\mathbb{A}^{i}$-bimodule map. We apply $\langle i\rangle_{*}$ and observe, using (4), that the same situation prevails in $\mathbb{A}$-bimod. So $\langle i\rangle_{*} g$ extends to an $\mathbb{A}$-bimodule map $h:\langle i\rangle_{*} M \rightarrow\langle i\rangle_{*} E$. Then (1) and (2) imply that $\langle i\rangle^{*} h$ extends $g$.

For the adjunction, suppose that $\eta \in \operatorname{Hom}_{\mathrm{A}-\mathrm{A}}\left(\mathbb{N},\langle i\rangle_{*} M\right)$. Then for $j \geq i$ we have $\eta^{i} T^{i j}=\eta^{j}$ and for $j \nsupseteq i$ we have $\eta^{j}=0$. So $\eta$ is completely determined by $\eta^{i} \in \operatorname{Hom}_{\mathbf{A}^{i}-\mathbb{A}^{i}}\left(\mathbb{N}^{i}, M\right)$. Conversely, any $\eta^{i} \in \operatorname{Hom}_{\mathbf{A}^{i}-\mathbb{A}^{i}}\left(\mathbb{N}^{i}, M\right)$ yields a map $\eta \in \operatorname{Hom}_{\mathrm{A}-\mathrm{A}}\left(\mathbb{N},\langle i\rangle_{*} M\right)$ by the formulae above.

We refer to $\langle i\rangle_{*}$ as the right inflation functor. A relative injective $\mathbb{A}$-bimodule having the form $\langle i\rangle_{*} E$ will be called a primitive (or $i$-primitive) relative injective.

PROOF OF THEOREM 1.1. It suffices to show that every $\mathbb{A}$-bimodule $\mathbb{M}$ has an allowable monomorphism $\mathbb{M} \mapsto \mathbb{E}$ to a relative injective $\mathbb{A}$-bimodule $\mathbb{E}$. Now, $\mathbb{A}^{i}$-bimod has enough relative injectives for each $i \in \mathscr{I}$. (This follows, essentially, from the fact that the forgetful functor $\mathbb{A}^{i}$-bimod $\rightarrow k$-bimod: $M \rightarrow|M|$ has a 
right adjoint, namely $V \leadsto \operatorname{Hom}_{k}\left(\mathbb{A}^{i} \otimes \mathbb{A}^{i}, V\right)$ (cf. [M, $\S \S I X .5$, IX.6].) Hence for each $i \in \mathscr{I}$ there is an allowable $\mathbb{A}^{i}$-bimodule monomorphism from $\mathbb{M}^{i}$ to a relative injective $\mathbb{A}^{i}$-bimodule $E_{i}$. Using the adjunction of the lemma we have allowable maps $\mathbb{M} \rightarrow\langle i\rangle_{*} E_{i}$ and, so, an allowable map $\mathbb{M} \rightarrow \mathbb{E}$ where $\mathbb{E}$ is the product in $\mathbb{A}$-bimod of $\left\{\langle i\rangle_{*} E_{i}\right\}$. As a product of relative injectives, $\mathbb{E}$ is a relative injective. Finally, since one of the components of $\mathbb{M}^{i} \rightarrow \mathbb{E}^{i}$ is $\mathbb{M}^{i} \mapsto \mathbb{E}_{i}$, each $\mathbb{M}^{i} \rightarrow \mathbb{E}^{i}$ is an allowable monomorphism and, so, $\mathbb{M} \rightarrow \mathbb{E}$ is an allowable monomorphism as well.

COROLLARY 1.3. A-bimod has enough relative injectives which are products of primitive relative injectives.

If $k$ is a field and $\mathscr{I}$ satisfies the descending chain condition then every relative injective $\mathbb{A}$-bimodule is a product of primitive relative injectives. Since we shall not use this fact we leave it as an exercise to the reader.

Observe that the functor $U_{1}: \mathbb{A}$-bimod $\rightarrow k$-bimod defined by $U_{1}(\mathbb{M})=\amalg\left|\mathbb{M}^{i}\right|$ preserves and reflects allowability. The proofs of Theorem 1.1 and Lemma 1.2 show that $U_{1}$ has a right adjoint, namely $V \leadsto \prod\langle i\rangle_{*}\left(\operatorname{Hom}_{k}\left(\mathbb{A}^{i} \otimes \mathbb{A}^{i}, V\right)\right)$, a product in $\mathbb{A}$-bimod. If $\mathcal{I}$ is infinite then $U_{1}$ does not preserve products and, consequently, cannot have a left adjoint.

The discussion thus far dualizes to yield, first, the definitions of relative projective and relative projective allowable resolution. Second, each restriction functor has a left adjoint, $\langle i\rangle_{!}: \mathbb{A}^{i}$-bimod $\rightarrow \mathbb{A}$-bimod, which is right exact (as is any left adjoint), preserves both allowability and relative projectivity, and is right inverse to $\langle i\rangle^{*}$. (However, it need not be exact.) It is defined as follows: $(\langle i\rangle ! M)^{h}=\mathbb{A}^{h} \otimes_{\mathrm{A}^{i}} M \otimes_{\mathrm{A}^{i}} \mathbb{A}^{h}$ if $h \leq i$ and $(\langle i\rangle ! M)^{h}=0$ if $h \$ i ;(\langle i\rangle ! M)^{j} \rightarrow(\langle i\rangle ! M)^{h}$ is $\varphi^{h j} \oplus \operatorname{Id}_{M} \oplus \varphi^{h j}$ if $h \leq j \leq i$ and is 0 otherwise. A primitive relative projective is a relative projective having the form $\langle i\rangle_{!} P$ for some $i \in \mathscr{I}$ and some (necessarily) relative projective $\mathbb{A}^{i}$ bimodule. Further, $\mathbb{A}$-bimod has enough relative projectives which are coproducts of primitive relative projectives. If $\mathscr{I}$ satisfies the ascending chain condition then these are the only relative projectives. (When $k$ is a field this follows from the argument in [GS1, §1]; for arbitrary $k$ it then follows by reducing modulo every maximal ideal of $k$.) Finally, the functor $U_{2}: \mathbb{A}$-bimod $\rightarrow k$-bimod defined by $U_{2}(\mathbb{M})=\prod\left|\mathbb{M}^{i}\right|$ preserves and reflects allowability and has a left adjoint, namely $V \leadsto \amalg\langle i\rangle_{!}\left(\mathbb{A}^{i} \otimes V \otimes \mathbb{A}^{i}\right)$, a coproduct in $\mathbb{A}$-bimod. (In [GS1, §6] we inadvertently identified the left adjoint of $U_{2}$ as a left adjoint to $U_{1}$.) If $\mathscr{I}$ is infinite then $U_{2}$ does not have a right adjoint; if $\mathscr{I}$ is finite then $U_{1}=U_{2}$.

We conclude this section with a discussion of the (relative) Yoneda cohomology bifunctor Ext $_{\mathbb{A}-\mathbf{A}}^{\bullet}(-,-)$ on $\mathbb{A}$-bimod, which, of course, is an instance of the general definition (cf. [M, $\S \S I I I .5$, XII.4]). We shall give a smattering of details to establish linguistic and notational conventions.

An allowable $n$-fold extension of $\mathbb{M}$ by $\mathbb{N}$ is an exact sequence, $\mathscr{E}: 0 \rightarrow \mathbb{M} \rightarrow$ $\mathbb{M}_{n} \rightarrow \cdots \rightarrow \mathbb{M}_{1} \rightarrow \mathbb{N} \rightarrow 0$, in which all maps are allowable. A map $\mathscr{E} \rightarrow \tilde{\mathscr{E}}$ of such extensions is a commutative diagram of $\mathbb{A}$-bimodule maps

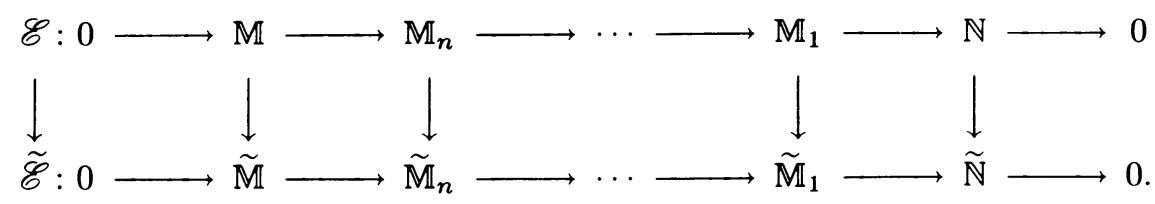


(N.B. The vertical maps need not be allowable.) The maps $\mathbb{M} \rightarrow \widetilde{\mathbb{M}}$ and $\mathbb{N} \rightarrow \widetilde{\mathbb{N}}$ are, respectively, the start and the end of $\mathscr{E} \rightarrow \widetilde{\mathscr{E}}$. We write $\mathscr{E} \equiv \widetilde{\mathscr{E}}$ to indicate a congruence of allowable extensions. Such a congruence can always be represented by a pair of maps $\mathscr{E} \leftarrow \mathscr{F} \rightarrow \tilde{\mathscr{E}}$, each starting and ending with an identity. We denote the 三-equivalence class of $\mathscr{E}$ by $[\mathscr{E}]$. As usual, $\operatorname{Ext}_{\mathrm{A}-\mathrm{A}}^{n}(\mathbb{N}, \mathbb{M})$ is the set of equivalence classes of allowable $n$-fold extensions of $\mathbb{M}$ by $\mathbb{N}$ if $n>0$ and $\operatorname{Ext}_{\mathbf{A}-\mathbf{A}}^{0}(\mathbb{N}, \mathbb{M})=\operatorname{Hom}_{\mathbf{A}-\mathbf{A}}(\mathbb{N}, \mathbb{M})$.

If $\nu: \mathbb{N}^{\prime} \rightarrow \mathbb{N}$ and $\mu: \mathbb{M} \rightarrow \mathbb{M}^{\prime}$ are $\mathbb{A}$-bimodule maps then we denote the pushout and pullback extensions-both of which are allowable-by $\mu \mathscr{E}=\mu \circ \mathscr{E}$ and $\mathscr{E} \nu=\mathscr{E} \circ \nu$. (For example, $\mathscr{E} \nu$ is $0 \rightarrow \mathbb{M} \rightarrow \mathbb{M}_{n} \rightarrow \cdots \rightarrow \mathbb{M}_{1} \nu \rightarrow \mathbb{N}^{\prime} \rightarrow 0$ where $\mathbb{M}_{1} \nu \rightarrow \mathbb{N}^{\prime}$ is the pullback of $\mathbb{M}_{1} \rightarrow \mathbb{N}$ along the map $\nu$.) Note that a map $\mathscr{E} \rightarrow \tilde{\mathscr{E}}$ which starts with $\mu$ and ends with $\nu$ induces a map $\mu \mathscr{E} \rightarrow \tilde{\mathscr{E}} \nu$ which is a congruence: $\mu \mathscr{E} \equiv \tilde{\mathscr{E}} \nu$. (This fact will be used repeatedly.) If $\mathscr{E}^{\prime}: 0 \rightarrow \mathbb{L} \rightarrow$ $\mathbb{L}_{m} \rightarrow \cdots \rightarrow \mathbb{M} \rightarrow 0$ is allowable then the Yoneda composite, or splice, $\mathscr{E}^{\prime} \circ \mathscr{E}$ is the allowable sequence $0 \rightarrow \mathbb{L} \rightarrow \mathbb{L}_{m} \rightarrow \cdots \rightarrow \mathbb{L}_{1} \rightarrow \mathbb{M}_{n} \rightarrow \cdots \rightarrow \mathbb{N} \rightarrow 0$ in which $\mathbb{L}_{1} \rightarrow \mathbb{M}_{n}$ is the composite $\mathbb{L}_{1} \rightarrow \mathbb{M} \rightarrow \mathbb{M}_{n}$. Splice, pushout, pullback, and composition of maps (in dimension 0 ) clearly preserve congruence; together they induce an associative $k$-bilinear operation, also denoted $\circ$, on $\operatorname{Ext}_{\mathrm{A}-\mathrm{A}}^{\bullet}(-,-)$. This means, in particular, that $\mathscr{F} \nu \circ \mathscr{E} \equiv \mathscr{F} \circ \nu \mathscr{E}$. Each allowable short exact sequence, $\mathscr{F}: 0 \rightarrow \mathbb{M}^{\prime} \rightarrow \mathbb{M} \rightarrow \mathbb{M}^{\prime \prime} \rightarrow 0$, induces a long exact sequence $\cdots \rightarrow \operatorname{Ext}_{\mathrm{A}-\mathrm{A}}^{n}\left(\mathbb{N}, \mathbb{M}^{\prime}\right) \rightarrow \operatorname{Ext}_{\mathrm{A}-\mathrm{A}}^{n}(\mathbb{N}, \mathbb{M}) \rightarrow \operatorname{Ext}_{\mathrm{A}-\mathrm{A}}^{n}\left(\mathbb{N}, \mathbb{M}^{\prime \prime}\right) \rightarrow \operatorname{Ext}_{\mathrm{A}-\mathrm{A}}^{n+1}\left(\mathbb{N}, \mathbb{M}^{\prime}\right) \rightarrow \cdots$ in which $\operatorname{Ext}_{\mathbf{A}-\mathbf{A}}^{n}\left(\mathbb{N}, \mathbb{M}^{\prime \prime}\right) \rightarrow \operatorname{Ext}_{\mathbf{A}-\mathbf{A}}^{n+1}\left(\mathbb{N}, \mathbb{M}^{\prime}\right)$ is defined by $[\mathscr{E}] \mapsto[\mathscr{F} \circ \mathscr{E}]$ and called the connecting homomorphism. The foreoging asserts that $\operatorname{Ext}_{\mathbf{A}-\mathbf{A}}^{\bullet}(\mathbb{N},-)$ is a relative $\delta$-functor for each $\mathbb{A}$-bimodule $\mathbb{N}$. Furthermore, $\operatorname{Ext}_{\mathbb{A}-\mathbf{A}}^{\bullet}(\mathbb{N},-)$ is universal, i.e. it has the following equivalent properties:

(1) $\operatorname{Ext}_{\mathbb{A}-\mathbf{A}}^{\bullet}(\mathbb{N}, \mathbb{E})=0$ if $n>0$ and $\mathbb{E}$ is a relative injective $\mathbb{A}$-bimodule;

(2) $\operatorname{Ext}_{\mathbf{A}-\mathbf{A}}^{\bullet}(\mathbb{N},-)=R^{\bullet} \operatorname{Hom}_{\mathbf{A}-\mathbf{A}}(\mathbb{N},-)$, the (relative) right-derived functors of $\operatorname{Hom}_{\mathbf{A}-\mathbf{A}}(\mathbb{N},-)$; and

(3) if $H^{\bullet}$ is a relative $\delta$-functor on $\mathbb{A}$-bimod and $f^{0}: \operatorname{Ext}_{\mathrm{A}-\mathrm{A}}^{0}(\mathbb{N},-) \rightarrow H^{0}$ is a natural transformation then $f^{0}$ has a unique extension to $f^{\bullet}: \operatorname{Ext}_{\mathbf{A}-\mathbf{A}}^{\bullet}(\mathbb{N},-) \rightarrow H^{\bullet}$. Likewise, $\operatorname{Ext}_{\mathbf{A}-\mathbf{A}}^{\bullet}(-, \mathbb{M})$ is a universal $\delta$-functor on $\mathbb{A}$-bimod.

Of course, in the classical case these definitions coincide with the usual ones. The foregoing can be formulated using arbitrary $n$-fold extensions, not merely allowable ones. The result is an "absolute" Yoneda cohomology. Historically, the absolute cohomology has been less useful than the relative one. Even so, it is worth noting that all of the lemmas, theorems, and proofs in $\S \S 1-5$ of this paper remain valid if the "relative" concepts are replaced by the corresponding "absolute" ones.

2. The diagram ring and the CCT. We shall associate to each diagram $\mathbb{A}$ over $\mathscr{I}$ a $k$-algebra $\mathbb{A}$ ! which we call the diagram ring. The most economical description of $\mathbb{A}$ ! is: $\mathbb{A} !=$ row-finite $\mathscr{I} \times \mathscr{I}$ matrices $\left(a_{i j}\right)$ with $a_{i j} \in \mathbb{A}^{i}$ if $i \leq j$ and $a_{i j}=0$ otherwise; the matrix product $\left(a_{i j}\right)\left(b_{i j}\right)=\left(c_{i j}\right)$ is computed as usual with the understanding that, for $h \leq i \leq j$, the summand $a_{h i} \cdot b_{i j}$ of $c_{h j}$ is interpreted as $a_{h i} \cdot b_{i j}=a_{h i} \varphi^{h i}\left(b_{i j}\right)$. For our purposes the following is a more convenient representation of $\mathbb{A}$ ! as a $k$-bimodule

$$
\mathbb{A} !=\prod_{i \in \mathscr{I}} \coprod_{j \geq i} \mathbb{A}^{i} \varphi^{i j}
$$


where the suffixes $\varphi^{i j}$ serve to distinguish distinct copies of $\mathbb{A}^{i}$ from one another. A "general" element of $\mathbb{A}^{i} \varphi^{i j}$ will be denoted $a^{i} \varphi^{i j}$. It is convenient to use (infinite) sum notation for elements of $\mathbb{A}$ !, so that the general element is written $\sum a^{i} \varphi^{i j}$. Multiplication in $\mathbb{A}$ ! is defined by (infinite) linearity and the rule

$$
\left(a^{h} \varphi^{h i}\right)\left(a^{j} \varphi^{j l}\right)= \begin{cases}a^{h} \varphi^{h i}\left(a^{j}\right) \varphi^{h l} & \text { if } i=j, \\ 0 & \text { otherwise. }\end{cases}
$$

Let $1_{i}$ be the unit element of $\mathbb{A}^{i}$. Since $\left(a^{h} \varphi^{h i}\right)\left(1_{i} \varphi^{i j}\right)=a^{h} \varphi^{h j}$ and $\left(1_{h} \varphi^{h i}\right)\left(a^{i} \varphi^{i j}\right)$ $=\varphi^{h i}\left(a^{i}\right) \varphi^{h j}$, we may safely abbreviate $1_{i} \varphi^{i j}$ to $\varphi^{i j}$. The maps $\varphi^{i j}$ are then formally elements of $\mathbb{A}$ ! and we have $\varphi^{h i} \varphi^{i j}=\varphi^{h j}$ (as before), but also $\varphi^{h i} \varphi^{j l}=0$ when $i \neq j$. The unit of $\mathbb{A}$ ! is $1=\sum \varphi^{i i}$, so $\left\{\varphi^{i i}\right\}$ is a set of orthogonal idempotents which sum to 1 . Note that $\mathbb{A}^{i} \varphi^{i i}$ is a subalgebra of $\mathbb{A}$ !, although its unit is not $1 \in \mathbb{A}$ ! but is rather $\varphi^{i i}$. The obvious $k$-bimodule map $\mathbb{A}^{i} \rightarrow \mathbb{A}^{i} \varphi^{i i}: a \mapsto a \varphi^{i i}$ is an isomorphism of (unital) $k$-algebras.

The diagram ring arose originally as the endomorphism ring of a projective generator, $\mathbb{G}$, in the category of left $\mathbb{A}$-modules, $(\mathbb{A}-\mathbb{k}$ )-bimod (cf., [GS1, $\S 10$ and GS6, §2].) Further, when $\mathscr{I}$ is finite, $\mathbb{G}$ is small and $\operatorname{Hom}_{\mathbb{A}}(\mathbb{G},-):(\mathbb{A}-\mathbb{k})$-bimod $\rightarrow$ $(\mathbb{A} !-k)$-bimod is an equivalence between $(\mathbb{A}-\mathbb{k})$-bimod and the category of left $\mathbb{A}$ !modules. This trivially gives a cohomology isomorphism. (In fact, $\operatorname{Hom}_{\mathbb{A}}(\mathbb{G},-)$ induces a cohomology isomorphism when $\mathbb{A}$ is a diagram over an arbitrary small category [GS6, §2].) However, it is not the type of isomorphism we seek. The first problem we encounter is that $\operatorname{Hom}_{\mathbb{A}}(\mathbb{G},-)$ does not carry the left $\mathbb{A}$-module $\mathbb{A}$ to the left $\mathbb{A}$ !-module $\mathbb{A}$ !, but rather to $\operatorname{Hom}_{\mathbb{A}}(\mathbb{G}, \mathbb{A})=\prod \mathbb{A}^{i} \varphi^{i i}$. Moreover, left $\mathbb{A}$-modules are of less interest to us than $\mathbb{A}$-bimodules. For it is the cohomology of $\mathbb{A}$-bimodules which captures information regarding singular diagram (of algebras) extensions of $\mathbb{A}$, deformations of $\mathbb{A}$, and (when $\mathbb{A}=\mathbb{k}$ ) simplicial cohomology of $\mathscr{I}$. $\mathrm{Ab}$ initio, it seems that a possible remedy is to replace $\mathbb{A}$-bimod by the (isomorphic) category of left $\mathbb{A}^{\mathrm{e}}$-modules where $\mathbb{A}^{\mathrm{e}}=\mathbb{A} \otimes \mathbb{A}^{\text {op }}$ is the "enveloping diagram." This is still unsatisfactory. Recall that our goals for such a cohomology isomorphism include: (1) it should yield an isomorphism between the simplicial cohomology $\operatorname{Ext}_{\mathbf{k}-\mathbf{k}}^{\bullet}(\mathbf{k}, \mathbf{k})=H^{\bullet}(\mathscr{I}, k)$ and the Hochschild cohomology $H^{\bullet}\left(R_{\mathbf{k}}, R_{\mathbf{k}}\right)$ of some $k$ algebra $R_{\mathbf{k}} ;(2)$ it should permit the reduction of questions about deformations and singular extensions of $\mathbb{A}$ to analogous questions about some $k$-algebra $R_{\mathrm{A}}$. As before, this means we wish to associate to each diagram $\mathbb{A}$ a $k$-algebra $R_{\mathbb{A}}$ and a functor $\left(\mathbb{A}^{\mathrm{e}}-\mathrm{k}\right)$-bimod $\rightarrow R_{\mathrm{A}^{-}}$-bimod which preserves relative Yoneda cohomology and carries the left $\mathbb{A}^{\mathrm{e}}$-module $\mathbb{A}$ to the $R_{\mathrm{A}}$-bimodule $R_{\mathrm{A}}$. Now, to be sure, $R_{\mathrm{A}}$-bimod is isomorphic to $\left(R_{\mathrm{A}}^{\mathrm{e}}-k\right)$-bimod. However the equivalence of categories we have in hand is $\left(A^{\mathrm{e}}-\mathbb{k}\right)$-bimod $\sim\left(\mathbb{A}^{\mathrm{e}}\right.$ !- $\left.\mathbf{k}\right)$-bimod and the latter category need not be equivalent to the category of left $R^{\mathrm{e}}$-modules for any $k$-algebra $R$. (For an example, set $\mathbb{A}=\operatorname{Id}_{k}: k \rightarrow k$ for a field $k$. Details appear in [GS6, §3].) In this vein it is worth noting, in particular, that $\mathbb{A}^{\mathrm{e}} ! \neq \mathbb{A} !$. The isomorphism between the category of $R$-bimodules and that of left $R^{\mathrm{e}}$-modules is quite useful. But it is not a panacea and its mere existence does not oblige one to use it. Indeed, as we have tried to illustrate above, there are times when it simply is not pertinent.

We wish to define a functor $!: \mathbb{A}$-bimod $\rightarrow \mathbb{A}$ !-bimod in such a way that the $\mathbb{A}$ bimodule $\mathbb{A}$ is carried to the $\mathbb{A}$ !-bimodule $\mathbb{A}$ !. This essentially forces the following definition of $\mathbb{M}$ ! for an $\mathbb{A}$-bimodule $\mathbb{M}$. As a $k$-bimodule $\mathbb{M} !=\prod_{i \in \mathcal{I}} \bigsqcup_{j \geq i} \mathbb{M}^{i} \varphi^{i j}$, 
the operation of $\mathbb{A}$ ! is defined by

$$
\begin{gathered}
\left(a^{h} \varphi^{h i}\right)\left(m^{i} \varphi^{i j}\right)=a^{h} T^{h i}\left(m^{i}\right) \varphi^{h j}, \\
\left(m^{h} \varphi^{h i}\right)\left(a^{i} \varphi^{i j}\right)=m^{h} \varphi^{h i}\left(a^{i}\right) \varphi^{h j}, \\
\left(a^{h} \varphi^{h i}\right)\left(m^{j} \varphi^{j l}\right)=0=\left(m^{h} \varphi^{h i}\right)\left(a^{j} \varphi^{j l}\right) \quad \text { if } i \neq j .
\end{gathered}
$$

Since $\mathbb{M}^{i} \varphi^{i i} \subset \mathbb{M}$ ! is closed under multiplication by $\mathbb{A}^{i} \varphi^{i i}$ and $\mathbb{A}^{i} \cong \mathbb{A}^{i} \varphi^{i i}$ we see that $\mathbb{M}^{i} \varphi^{i i}$ is naturally an $\mathbb{A}^{i}$-bimodule and that $\mathbb{M}^{i} \rightarrow \mathbb{M}^{i} \varphi^{i i}: m \mapsto m \varphi^{i i}$ is an $\mathbb{A}^{i}$-bimodule isomorphism.

If $\eta \in \operatorname{Hom}_{\mathbb{A}-\mathbb{A}}(\mathbb{N}, \mathbb{M})$ define $\eta ! \in \operatorname{Hom}_{\mathbb{A} !-\mathbb{A} !}(\mathbb{N} !, \mathbb{M} !)$ by $\eta !\left(n^{i} \varphi^{i j}\right)=\eta^{i}\left(n^{i}\right) \varphi^{i j}$. At this juncture it is worth noting that, as will be discussed below, ! is an equivalence if and only if $\mathscr{I}$ is discrete. (Also see the remarks following Theorem 3.9.) We now prove the dimension zero case of the CCT, namely

THEOREM 2.1. !: $\mathbb{A}$-bimod $\rightarrow \mathbb{A}$ !-bimod preserves allowability and is a full exact embedding.

ProOF. We prove only that ! is full, the other claims being trivial. Let $\mathbb{M}$ and $\mathbb{N}$ be $\mathbb{A}$-bimodules and $g: \mathbb{N} ! \rightarrow \mathbb{M}$ ! be an $\mathbb{A}$ !-bimodule map. Since $g\left(n^{i} \varphi^{i i}\right)=$ $g\left(n^{i} \varphi^{i i}\right) \varphi^{i j}$, it is clear that $g$ is determined by its restrictions $g^{i}: \mathbb{N}^{i} \varphi^{i i} \rightarrow \mathbb{M}$ !. Furthermore, for $n \in \mathbb{N}^{i}$ we have $n \varphi^{i i}=\varphi^{i i}\left(n \varphi^{i i}\right) \varphi^{i i}$ and, consequently, $g^{i}\left(n^{i} \varphi^{i i}\right)=$ $\varphi^{i i} g\left(n^{i} \varphi^{i i}\right) \varphi^{i i} \in \varphi^{i i} \mathbb{M} ! \varphi^{i i}=\mathbb{M}^{i} \varphi^{i i}$. Now, trivially, $g^{i}$ is an $\mathbb{A}^{i}$-bimodule map $\mathbb{N}^{i} \varphi^{i i} \rightarrow \mathbb{M}^{i} \varphi^{i i}$. Using the remarks above the theorem we define $\hat{g}^{i}: \mathbb{N}^{i} \rightarrow \mathbb{M}^{i}$ by $\hat{g}^{i}(n) \varphi^{i i}=g^{i}\left(n \varphi^{i i}\right)$. These maps comprise an $\mathbb{A}$-bimodule map $\hat{g}: \mathbb{N} \rightarrow \mathbb{M}$ if and only if $\hat{g}^{i} \circ \mathbb{N}(i j)=\mathbb{M}(i j) \circ \hat{g}^{j}$ for all $i \leq j$. To see that this is so, observe that for $n \in \mathbb{N}^{j}$ we have both

$$
\begin{aligned}
\varphi^{i j} g\left(n \varphi^{j j}\right) & =g\left(\varphi^{i j} n \varphi^{j j}\right)=g\left(\mathbb{N}(i j)(n) \varphi^{i j}\right) \\
& =g\left(\mathbb{N}(i j)(n) \varphi^{i i}\right) \varphi^{i j}=\hat{g}^{i}(\mathbb{N}(i j)(n)) \varphi^{i j}
\end{aligned}
$$

and $\varphi^{i j} g\left(n \varphi^{j j}\right)=\varphi^{i j} \hat{g}^{j}(n) \varphi^{j j}=\mathbb{M}(i j)\left(\hat{g}^{j}(n)\right) \varphi^{i j}$. Now right multiplication by $\varphi^{i j}$ is a $k$-bimodule isomorphism $\mathbb{M}^{i} \rightarrow \mathbb{M}^{i} \varphi^{i j}$. Hence the last two equations show that $\hat{g}(\mathbb{N}(i j)(n))=\mathbb{M}(i j)\left(\hat{g}^{j}(n)\right)$, as required. That $\eta \mapsto \eta$ ! and $g \mapsto \hat{g}$ are inverses is routine.

Thus, $\operatorname{Hom}_{\mathrm{A}-\mathrm{A}}(-,-) \rightarrow \operatorname{Hom}_{\mathbf{A} !-\mathrm{A} !}(-,-): \eta \mapsto \eta !$ is a natural isomorphism. Since ! is exact, it follows that $\operatorname{Ext}_{\mathbf{A} !-\mathrm{A} !}^{\bullet}(-!,-!)$ is a relative $\delta$-functor (in each argument) on $\mathbb{A}$-bimod. Hence, the universality of $\operatorname{Ext}_{\mathbb{A}-\mathbb{A}}^{\bullet}(-,-)$ guarantees that $\eta \mapsto \eta$ ! has a unique extension $\omega^{\bullet}$ : $\operatorname{Ext}_{\mathrm{A}-\mathrm{A}}^{\bullet}(-,-) \rightarrow \operatorname{Ext}_{\mathrm{A} !-\mathrm{A} !}^{\bullet}(-! .-!)$. Now, in an obvious way, every allowable extension $\mathscr{E}$ in $\mathbb{A}$-bimod gives rise to an allowable extension $\mathscr{E}$ ! in A! -bimod. The correspondence $\mathscr{E} \mapsto \mathscr{E}$ ! clearly preserves equivalence, pushouts, and splices and is a natural transformation which extends $\eta \mapsto \eta !$. So it must be $\omega^{\bullet}$. A priori, $\omega^{\bullet}$ need not be an isomorphism - there may well be extensions connecting $\mathbb{M}$ ! and $\mathbb{N}$ ! in $\mathbb{A}$ !-bimod which do not have the form $\mathscr{E}$ !. Nevertheless, we shall prove

THE SPECIAL COHOMOLOGY COMPARISON THOEREM (CCT). If $\mathbb{A}$ is an arbitrary diagram over an arbitrary poset then $\omega^{\bullet}: \operatorname{Ext}_{\mathbf{A}-\mathbf{A}}^{\bullet}(-,-) \rightarrow \operatorname{Ext}_{\mathbf{A} !-\mathbf{A} !}^{\bullet}(-!,-!)$ is an isomorphism.

If $\mathscr{I}$ is discrete $(i \leq j \Rightarrow i=j)$ then $\mathbb{A} !=\prod \mathbb{A}^{i} \varphi^{i i}$ and the CCT follows from the obvious equivalences of categories $\mathbb{A}$ !-bimod $\sim \prod \mathbb{A}^{i} \varphi^{i i}$-bimod $\sim \mathbb{A}$-bimod. In this case ! preserves all relative injectives and projectives. Indeed the CCT would 
be trivial in general if ! preserved either enough relative injectives or enough relative projectives; unfortunately, it does neither. In fact it fails in a rather spectacular way: a nontrivial $i$-primitive injective is preserved by ! if and only if $i$ is maximal in $\mathscr{F}$; if $k$ is a field and $\mathscr{I}$ has the descending chain condition then these (and products of them) are the only relative injectives! preserves. We shall prove this in $\S 5$. (Also see [GS1, §11 and GS3, §3].) In particular, if $\mathscr{J}$ is not discrete then ! is not an equivalence. We conjecture, however, that it has both adjoints. (If so, they are necessarily inexact.) We should also remark that the CCT is genuinely a theorem about bimodules and only bimodules. Specifically, with obvious modifications to (2.3), ! may be defined for left $\mathbb{A}$-modules and is then an embedding of left module categories, $(\mathbb{A}-k)$-bimod $\rightarrow(\mathbb{A} !-k)$-bimod, which carries $\mathbb{A}$ to $\mathbb{A}$ ! However, this embedding rarely preserves (relative) Yoneda cohomology. Indeed, more generally, if $\mathcal{I}$ is homologically nontrivial then there does not exist an algebra $R$ and a functor $(\mathbb{A}-\mathbb{k})$-bimod $\rightarrow(R-k)$-bimod which carries $\mathbb{A}$ to $R$ and preserves (relative Yoneda cohomology [GS3, §3].

Now let $\mathbb{E}$ be a primitive relative injective $\mathbb{A}$-bimodule. While $\mathbb{E}$ ! need not be a relative injective $\mathbb{A}$ !-bimodule, it will be "acyclic enough" to imply the CCT. Specifically, the next two sections will be devoted to proving

THE (FIRST) ACYCLICITY THEOREM. If $\mathbb{E}$ and $\mathbb{N}$ are $\mathbb{A}$-bimodules and $\mathbb{E}$ is a primitive relative injective then $\mathbb{E}$ ! is a relative $\operatorname{Hom}_{\mathbb{A} !-\mathbb{A} !}(\mathbb{N} !,-)$-acyclic bimodule; that is, $\operatorname{Ext}_{\mathbb{A} !-\mathbf{A} !}^{n}(\mathbb{N} !, \mathbb{E} !)=\left(R^{n} \operatorname{Hom}_{\mathbb{A} !-\mathbb{A} !}(\mathbb{N} !,-)\right)(\mathbb{E} !)=0$ for $n>0$.

The relevance of the Acyclicity Theorem derives from

THEOREM 2.2. The CCT is equivalent to the Acyclicity Theorem.

PROOF. In the presence of the CCT we have $\operatorname{Ext}_{\mathbb{A} !-\mathbb{A}}^{n}(\mathbb{N} !, \mathbb{E} !) \cong \operatorname{Ext}_{\mathbb{A}-\mathbb{A}}^{n}(\mathbb{N}, \mathbb{E})=0$ for $n>0$. This is half of the equivalence. Before proving the other half we citewithout proof-a general though quite standard result. Suppose $\mathscr{A}$ and $\mathscr{B}$ are abelian categories, $\mathscr{A}$ has enough (relative) injectives, $F: \mathscr{A} \rightarrow \mathscr{B}$ is a covariant left exact functor, and $0 \rightarrow A \rightarrow E_{\bullet}$ is an (allowable) resolution of $A \in \mathscr{A}$ by (relative) $F$-acyclic objects in $\mathscr{A}$. Then $\left(R^{\bullet} F\right)(A)=H\left(F\left(E_{\bullet}\right)\right)$; that is, (relative) cohomology can be computed using (relative) acyclic (allowable) resolutions (cf. [CE, §XVII.3 and Gr, Theorem 2.4.1, Remark 3]).

Now assume the Acyclicity Theorem holds. Then, since a product of acyclic objects is acyclic, $\mathbb{E}$ ! is $\operatorname{Hom}_{\mathbb{A} !-\mathbb{A} !}(\mathbb{N} !,-)$-acyclic whenever $\mathbb{E}$ is a product of primitive injective $\mathbb{A}$-bimodules. Let $\mathbb{M}$ be an $\mathbb{A}$-bimodule and let $0 \rightarrow \mathbb{M} \rightarrow \mathbb{E}$. be a relative injective allowable resolution of $\mathbb{M}$ in which every $\mathbb{E}_{n}$ is a product of primitive relative injective $\mathbb{A}$-bimodules. (Such resolutions are guaranteed by Corollary 1.3.) We then have

$$
\begin{aligned}
\operatorname{Ext}_{\mathbb{A}-\mathbb{A}}^{\bullet}(\mathbb{N}, \mathbb{M}) & =H\left(\operatorname{Hom}_{\mathbb{A}-\mathbb{A}}\left(\mathbb{N}, \mathbb{E}_{\bullet}\right)\right)=H\left(\operatorname{Hom}_{\mathbb{A} !-\mathbb{A} !}(\mathbb{N} !, \mathbb{E} !)\right) \\
& =\left(R^{\bullet} \operatorname{Hom}_{\mathbb{A} !-\mathbb{A} !}(\mathbb{N} !,-)\right)(\mathbb{M} !)=\operatorname{Ext}_{\mathbb{A} !-\mathbb{A} !}^{\bullet}(\mathbb{N} !, \mathbb{M} !) .
\end{aligned}
$$

The second equality follows from Theorem 2.1; the third follows from the Acyclicity Theorem and the general remarks above; the other two are simply the assertions that $\operatorname{Ext}_{\mathbb{A}-\mathbb{A}}^{\bullet}(\mathbb{N},-)$ and $\operatorname{Ext}_{\mathbb{A} !-\mathbf{A} !}^{\bullet}(\mathbb{N} !,-)$ are relative right-derived functors.

One can, of course, formulate the concepts of diagrams and diagram rings over an arbitrary small category c: for diagrams simply replace $\mathscr{I}$ by $\mathfrak{c}$ throughout $\S 1$; for diagram rings replace $\amalg \mathbb{A}^{i} \varphi^{i j}$ by $\amalg \mathbb{A}^{i} \varphi^{v}$ where the latter coproduct is taken 
over the set of maps $v$ with domain $i$. It is natural then to ask whether the CCT holds in this generality. It does not. For example, suppose that $c$ is a nontrivial group and $\mathbb{k}$ is the constant diagram on $\mathfrak{c}$ defined by $k^{i}=\mathbb{k}$ for all $i$. Then $\mathbb{k} !=k[\mathfrak{c}]$, the group ring. But $\operatorname{Hom}_{\mathbf{k}-\mathbf{k}}(\mathbb{k}, \mathbb{k})=k$ while, if $\mathfrak{c}$ is abelian, $\operatorname{Hom}_{k[\mathfrak{c}]}(k[\mathfrak{c}], k[\mathfrak{c}])$ is $k[\mathrm{c}]$. Thus, the dimension zero case of the CCT, namely Theorem 2.1, can fail if $\mathfrak{c}$ is not a poset. In [GS5, 6] we rectify this situation in the following way. We define a functor $\mathfrak{c} \leadsto \mathfrak{c}^{\prime}$ from the category of small categories to itself. We call $\mathfrak{c}^{\prime}$ the barycentric subdivision of $\mathfrak{c}$ since that is precisely what it is when $\mathfrak{c}$ is a poset. We also define a natural transformation from the subdivision functor to the identity functor. Every diagram $\mathbb{A}$ over $\mathfrak{c}$ then has a subdivision $\mathbb{A}^{\prime}$ (over $\mathfrak{c}^{\prime}$ ) which is obtained by composing $\mathbb{A}$ with the functor $\mathfrak{c}^{\prime} \rightarrow \mathfrak{c}$. Similarly, there is a subdivision functor $\mathbb{A}$-bimod $\rightarrow \mathbb{A}^{\prime}$-bimod. We prove that the latter functor induces an isomorphism of (relative) Yoneda cohomologies and that the second subdivision, $\mathfrak{c}^{\prime \prime}$, is always a poset. Hence, we obtain the General Cohomology Comparison Theorem: the (composed) functor $\mathbb{A}$-bimod $\rightarrow \mathbb{A}^{\prime \prime}$ !-bimod induces an isomorphism of (relative) Yoneda cohomologies. The cohomology of an arbitrary diagram over an arbitrary small category thus always reduces to the cohomology of an algebra.

3. Reduction to the second acyclicity theorem. Our objective in this section is to prove a theorem that significantly reduces the task posed by the Acyclicity Theorem, which requries us to compute $\operatorname{Ext}_{\mathbf{A} !-\mathbf{A} !}^{\bullet}(\mathbb{N} !, \mathbb{E}$ !) for an arbitrary primitive relative injective $\mathbb{A}$-bimodule $\mathbb{E}$. Now $\mathbb{E}=\langle i\rangle_{*} E$ for some $i \in \mathscr{I}$ and some relative injective $\mathbb{A}^{i}$-bimodule $E$. We shall show that, in a sense soon to be made precise, $\operatorname{Ext}_{\mathbb{A} !-\mathrm{A} !}^{\bullet}\left(\mathbb{N} !,\left(\langle i\rangle_{*}-\right) !\right)$ is "determined over" the subposet $i / \mathscr{I}=\{j \geq i\}$. First we review our notational conventions: + will be used for direct sum in $k$-bimod; otherwise we use $\oplus$; also, $\Pi$ and $\amalg$, when used without comment, indicate product and coproduct in $k$-bimod only.

A filter in a poset $\mathscr{I}$ is a subset $\mathscr{J} \subset \mathscr{I}$ with the property: if $j \in \mathscr{J}$ and $i \geq j$ then $i \in \mathscr{J}$. Every element $i \in \mathscr{I}$ generates a principle filter, namely $i / \mathscr{I}=\{j \geq i\}$. If $\mathbb{A}: \mathscr{J}^{\text {op }} \rightarrow k$-alg is a diagram then the restriction of $\mathbb{A}$ to a subposet. $\mathscr{J} \subset \mathscr{I}$ is the diagram $\langle\mathscr{J}\rangle^{*} \mathbb{A}: \mathscr{J}^{\text {op }} \rightarrow \mathscr{I}$ op $\rightarrow k$-alg. There is then likewise a restriction functor $\mathbb{A}$-bimod $\rightarrow\left(\langle\mathcal{F}\rangle^{*} \mathbb{A}\right)$-bimod: $\mathbb{M} \mapsto\langle\mathcal{J}\rangle^{*} \mathbb{M}$. We elide the proof of the following lemma since it is similar to the proof of Lemma 1.2.

LEMMA 3.1. Let $\mathscr{J} \subset \mathcal{I}$ be a filter and let $\mathbb{A}$ be a diagram over $\mathscr{I}$.

(1) The restriction functor $\langle\mathscr{J}\rangle^{*}: \mathbb{A}$-bimod $\rightarrow\left(\langle\mathscr{J}\rangle^{*} \mathbb{A}\right)$-bimod is exact and preserves allowability.

(2) $\langle\mathscr{J}\rangle^{*}$ has a right inverse $\langle\mathscr{F}\rangle_{*}:\left(\langle\mathscr{J}\rangle^{*} \mathbb{A}\right)$-bimod $\rightarrow \mathbb{A}$-bimod defined by: $\left(\langle\mathcal{J}\rangle_{*} \mathbb{M}\right)^{i}=M^{i}$ if $i \in \mathscr{J}$ and $\left(\langle\mathcal{J}\rangle_{*} \mathbb{M}\right)^{i}=0$ otherwise; $\left(\langle\mathcal{J}\rangle_{*} \mathbb{M}\right)^{i} \rightarrow\left(\langle\mathcal{J}\rangle_{*} \mathbb{M}\right)^{h}$ is 0 unless $h \leq i$ and $h \in \mathcal{J}$, in which case it is $\mathbb{M}^{i} \rightarrow \mathbb{M}^{h}$.

(3) $\langle\mathscr{J}\rangle_{*}$ is right adjoint to $\langle\mathcal{F}\rangle^{*}$.

(4) $\langle\mathscr{J}\rangle_{*}$ is exact and preserves both allowability and relative injectivity.

(5) $\langle\mathscr{J}\rangle_{*} \mathbb{E}$ is a relative injective $\mathbb{A}$-bimodule if and only if $\mathbb{E}$ is a relative injective $\left(\langle\mathcal{J}\rangle^{*} \mathbb{A}\right)$-bimodule.

Note that $\langle\mathscr{J}\rangle_{*}$ is just "extension by 0 ". For diagrams it is now easy to see the sense in which $\operatorname{Ext}_{\mathrm{A}-\mathrm{A}}^{\bullet}\left(\mathbb{N},\langle i\rangle_{*} M\right)$ is "determined over" $i / \mathscr{I}$. Indeed, if $\mathscr{J}$ is any filter in $\mathscr{I}$ and $\mathbb{M}$ is a $\left(\langle\mathscr{J}\rangle^{*} \mathbb{A}\right)$-bimodule then $\operatorname{Ext}_{\mathbb{A}-\mathbb{A}}^{\bullet}\left(\mathbb{N},\langle\mathscr{J}\rangle_{*} \mathbb{M}\right)$ is "determined 
over" $\mathscr{J}$. Specifically, observe that -in an obvious way-restriction gives a natural transformation $\operatorname{Ext}_{\mathbb{A}-\mathbb{A}}^{\bullet}\left(\mathbb{N},\langle\mathscr{J}\rangle_{*}-\right) \rightarrow \operatorname{Ext}_{\left(\langle\mathcal{J}\rangle^{*} \mathbb{A}\right)-\left(\langle\mathcal{J}\rangle^{*} \mathbb{A}\right)}\left(\langle\mathscr{J}\rangle^{*} \mathbb{N},-\right):[\mathscr{E}] \mapsto$ $\left[\langle\mathscr{J}\rangle^{*} \mathscr{E}\right]$. (Note that this requires $\langle\mathscr{J}\rangle^{*}\langle\mathscr{J}\rangle_{*}=$ the identity functor on $\left(\langle\mathscr{J}\rangle^{*} \mathbb{A}\right.$ )bimod.) Then we have

THEOREM 3.2. For each $\mathbb{A}$-bimodule $\mathbb{N}$ the natural transformations

$$
\operatorname{Ext}_{\mathbb{A}-\mathbb{A}}^{\bullet}\left(\mathbb{N},\langle\mathscr{J}\rangle_{*}-\right) \rightarrow \operatorname{Ext}_{\left(\langle\mathscr{J}\rangle^{*} \mathbb{A}\right)-\left(\langle\mathscr{J}\rangle^{*} \mathbb{A}\right)}^{\bullet}\left(\langle\mathscr{J}\rangle^{*} \mathbb{N},-\right)
$$

and

$$
\operatorname{Ext}_{\mathbb{A}-\mathbb{A}}^{\bullet}\left(\mathbb{N},\langle i\rangle_{*}-\right) \rightarrow \operatorname{Ext}_{\mathbb{A}^{i}-\mathbb{A}^{i}}^{\bullet}\left(\mathbb{N}^{i},-\right):[\mathscr{E}] \mapsto\left[\langle i\rangle^{*} \mathscr{E}\right]
$$

are isomorphisms.

PROOF. Part 4 of Lemma 3.1 implies that $\operatorname{Ext}_{\mathbb{A}-\mathbb{A}}^{\bullet}\left(\mathbb{N},\langle\mathscr{J}\rangle_{*}-\right)$ is a relative $\delta$ functor on $\left(\langle\mathscr{J}\rangle^{*} \mathbb{A}\right)$-bimod which vanishes on relative injectives and, so, is universal. The same is true of $\operatorname{Ext}_{\left(\langle\mathscr{g}\rangle^{*} \mathbb{A}\right)-\left(\langle\mathscr{f}\rangle^{*} \mathbb{A}\right)}\left(\langle\mathscr{J}\rangle^{*} \mathbb{N},-\right)$. The adjunction provides an isomorphism $\operatorname{Hom}_{\mathbb{A}-\mathbb{A}}\left(\mathbb{N},\langle\mathscr{J}\rangle_{*}-\right) \rightarrow \operatorname{Hom}_{\left(\langle\mathscr{J}\rangle^{*} \mathbb{A}\right)-\left(\langle\mathscr{J}\rangle^{*} \mathbb{A}\right)}\left(\langle\mathscr{J}\rangle^{*} \mathbb{N},-\right)$ between these $\delta$-functors in dimension zero. Hence it extends uniquely to a map $\operatorname{Ext}_{\mathbb{A}-\mathbb{A}}^{\bullet}\left(\mathbb{N},\langle\mathscr{J}\rangle_{*}-\right) \rightarrow \operatorname{Ext}_{\left(\langle\mathscr{J}\rangle^{*} \mathbb{A}\right)-\left(\langle\mathscr{J}\rangle^{*} \mathbb{A}\right)}\left(\langle\mathscr{J}\rangle^{*} \mathbb{N},-\right)$ which is then necessarily an isomorphism. But $[\mathscr{E}] \mapsto\left[\langle\mathscr{J}\rangle^{*} \mathscr{E}\right]$ extends the adjunction map and, so, is that isomorphism. The second claim follows from Lemma 1.2 in a similar fashion.

We shall use Lemma 3.1 again in $§ 5$. Now, granting the CCT for a moment, the first of these isomorphisms translates to an isomorphism $\left.\operatorname{Ext}_{\mathbb{A} !-\mathbb{A} !}^{\bullet}\left(\mathbb{N} !,\langle\mathscr{J}\rangle_{*} \mathbb{M}\right) !\right) \cong$ $\operatorname{Ext}_{\left(\langle\mathscr{f}\rangle^{*} \mathbb{A}\right) !-\left(\langle\mathscr{f}\rangle^{*} \mathbb{A}\right) !}\left(\left(\langle\mathcal{J}\rangle^{*} \mathbb{N}\right) !, \mathbb{M} !\right)$. We shall derive this as Theorem 3.7 and it will be the linchpin of our reduction of the Acyclicity Theorem. (The second isomorphism of Theorem 3.2 will be discussed after Theorem 3.7.) The path to Theorem 3.7 is not quite as straight as that to Theorem 3.2. The obstruction is the failure of the forgetful functor $\left(\langle\mathcal{F}\rangle^{*} \mathbb{A}\right)$ !-bimod $\rightarrow \mathbb{A}$ !-bimod provided by Theorem 3.3 (below), to preserve relative injectives. We begin by collecting some elementary observations which will also be of use in $\S 4$.

THEOREM 3.3. (1) $e=\sum_{j \in \mathcal{J}} \varphi^{j j}$ is an idempotent.

(2) $\mathbb{A} ! e$ and $(1-e) \mathbb{A}$ ! are two-sided ideals in $\mathbb{A}$ !.

(3) If $\mathfrak{a}$ is an $\mathbb{A}$ !-bimodule then $(1-e) \mathfrak{a}$ and $\mathfrak{a} e$ are $\mathbb{A}$ !-subbimodules of $\mathfrak{a}$.

(4) There is a k-algebra isomorphism $\mathbb{A} ! /(1-e) \mathbb{A} ! \cong\left(\langle\mathscr{J}\rangle^{*} \mathbb{A}\right)$ !.

(5) The forgetful functors induced by $\mathbb{A} ! \rightarrow\left(\langle\mathscr{J}\rangle^{*} \mathbb{A}\right)$ ! are exact and preserve allowability.

PROOF. Part 1 is obvious and (3) is an immediate consequence of (2). For the latter observe the following $k$-bimodule decompositions:

$$
\begin{gathered}
e \mathbb{A} !=\prod_{i \in \mathcal{F}} \coprod_{j \geq i} \mathbb{A}^{i} \varphi^{i j} \quad \text { and } \mathbb{A} ! e=\prod_{i \in \mathscr{J}} \coprod_{\substack{j \geq i \\
j \in \mathcal{J}}} \mathbb{A}^{i} \varphi^{i j}, \\
\mathbb{A} !(1-e)=\prod_{i \in \mathscr{I}} \coprod_{\substack{j \geq i \\
j \notin \mathscr{J}}} \mathbb{A}^{i} \varphi^{i j} \quad \text { and } \quad(1-e) \mathbb{A} !=\prod_{i \notin \mathscr{J}} \coprod_{j \geq i} \mathbb{A}^{i} \varphi^{i j} .
\end{gathered}
$$

Hence, $e \mathbb{A} ! \subset \mathbb{A} ! e$ and, since $\mathcal{J}$ is a filter, $\mathbb{A} !(1-e) \subset(1-e) \mathbb{A}$ !, which yields (2). Next, the description of $(1-e) \mathbb{A}$ ! given above, when combined with $(2.1)$, provides an obvious $k$-bimodule isomorphism $\mathbb{A} ! /(1-e) \mathbb{A} ! \rightarrow\left(\langle\mathcal{J}\rangle^{*} \mathbb{A}\right)$ !, which is 
easily checked to be a $k$-algebra isomorphism. The last part of the theorem is evident.

If $\mathfrak{a}$ is an $\mathbb{A}$ !-bimodule, then $\overline{\mathfrak{a}}=\mathfrak{a} /(1-e) \mathfrak{a}$ is an $\mathbb{A}$ !-bimodule and $(1-e) \overline{\mathfrak{a}}=$ 0 . Hence $\overline{\mathfrak{a}}$ has a natural structure as a $\left(\langle\mathscr{J}\rangle^{*} \mathbb{A}\right.$ !- $\left.\mathbb{A} !\right)$-bimodule and $\mathfrak{a} \rightsquigarrow \overline{\mathfrak{a}}=$ $\mathfrak{a} /(1-e) \mathfrak{a}$ defines a functor $\mathbb{A} !-b i m o d \rightarrow\left(\left(\langle\mathscr{J}\rangle^{*} \mathbb{A}\right) !-\mathbb{A} !\right)$-bimod. Of course, $\overline{\mathfrak{a}}$ can be viewed, through $\mathbb{A} ! \rightarrow\left(\langle\mathscr{J}\rangle^{*} \mathbb{A}\right)$ !, as an $\mathbb{A}$ !-bimodule again and the two $\mathbb{A}$ !-bimodule structures on $\overline{\mathfrak{a}}$ are identical. Similarly, a $e$ is an $\mathbb{A}$ !-bimodule and $(\mathfrak{a} e)(1-e)=0$. Thus $\mathfrak{a} e$ may be construed as an $\left(\mathbb{A} !-\left(\langle\mathscr{J}\rangle^{*} \mathbb{A}\right)\right.$ !)-bimodule and $\mathfrak{a} \rightsquigarrow \mathfrak{a} e$ defines a functor $\mathbb{A}$ !-bimod $\rightarrow\left(\mathbb{A} !-\left(\langle\mathscr{J}\rangle^{*} \mathbb{A}\right)\right.$ !)-bimod. The $\mathbb{A}$ !-bimodule structure ae then acquires through $\mathbb{A} ! \rightarrow\left(\langle\mathscr{J}\rangle^{*} \mathbb{A}\right)$ ! is identical to its structure as a subbimodule of $\mathfrak{a}$. (These observations will be used in $\S 4$ also.) In view of the comments above, we may combine these constructions to obtain a functor $\mathbb{A}$ !-bimod $\rightarrow\left(\langle\mathscr{J}\rangle^{*} \mathbb{A}\right)$ !-bimod: $\mathfrak{a} \rightsquigarrow$ $\overline{\mathfrak{a}} e$. The latter functor is the essential tool needed to replicate the first isomorphism of Theorem 3.2 over diagram rings. That $\mathfrak{a} \leadsto \overline{\mathfrak{a}} e$ is exact and preserves allowability follows immediately from

LEMMA 3.4. $\mathfrak{a} \rightsquigarrow \overline{\mathfrak{a}}=\mathfrak{a} /(1-e) \mathfrak{a}$ and $\mathfrak{a} \rightsquigarrow \mathfrak{a} e$ are exact functors which preserve allowability.

Proof. If $g \in \operatorname{Hom}_{\mathbb{A} !-\mathbb{A} !}(\mathfrak{a}, \mathfrak{b})$ then $g(e \mathfrak{a})=e g(\mathfrak{a}) \subset e \mathfrak{b}$. Moreover, there is a $k$ bimodule decomposition $\mathfrak{a}=(1-e) \mathfrak{a}+e \mathfrak{a}$. Hence, an allowable exact sequence $\mathscr{E}$ of A!-bimodules, when viewed in $k$-bimod, decomposes as $\mathscr{E}=(1-e) \mathscr{E}+e \mathscr{E}$ and both summands are split exact. It follows that the quotient sequence $\overline{\mathscr{E}}=\mathscr{E} /(1-e) \mathscr{E}$ is isomorphic in $k$-bimod to $e \mathscr{E}$, which means that $\mathfrak{a} \rightsquigarrow \overline{\mathfrak{a}}$ is exact and preserves allowability. The claims for $\mathfrak{a} \leadsto \mathfrak{a} e$ are established similarly.

A routine calculation shows that $\mathfrak{a} \rightsquigarrow \overline{\mathfrak{a}}$ is the left adjoint to the forgetful functor $\left(\left(\langle\mathcal{J}\rangle^{*} \mathbb{A}\right)\right.$ !- $\left.\mathbb{A} !\right)$-bimod $\rightarrow \mathbb{A}$ !-bimod. However, $\mathfrak{a} \rightsquigarrow \overline{\mathfrak{a}} e$ is not the left adjoint to $\left(\langle\mathscr{J}\rangle^{*} \mathbb{A}\right)$ !-bimod $\rightarrow \mathbb{A}$ !-bimod. On the other hand, it is a right inverse to the latter forgetful functor: viewing $\mathfrak{c} \in\left(\langle\mathscr{J}\rangle^{*} \mathbb{A}\right)$ !-bimod as an $\mathbb{A}$ !-bimodule we have $\overline{\mathfrak{c}} e=\mathfrak{c}$. Hence the functor $\mathfrak{a} \rightsquigarrow \overline{\mathfrak{a}} e$ provides for each $\mathbb{A}$ !-bimodule $\mathfrak{b}$ and each $\left(\langle\mathscr{J}\rangle^{*} \mathbb{A}\right)$ !bimodule $\mathfrak{c}$ a map

$$
\operatorname{Hom}_{\mathbb{A} !-A}(\mathfrak{b}, \mathfrak{c}) \rightarrow \operatorname{Hom}_{\left(\langle\mathcal{f}\rangle^{*} \mathbb{A}\right) !-\left(\langle\mathcal{f}\rangle^{*} \mathbb{A}\right) !}(\overline{\mathfrak{b}} e, \mathfrak{c}):\left.f \mapsto \bar{f}\right|_{\bar{b} e},
$$

which we claim is a monomorphism. Specifically, if $\left.\bar{f}\right|_{\bar{b} e}=0$ then, as is easily confirmed, $f(e \mathfrak{b} e)=0$. Since $f((1-e) \mathfrak{b}) \subset(1-e) \mathfrak{c}=0$ and $f(\mathfrak{b}(1-e)) \subset \mathfrak{c}(1-e)=0$, this establishes the claim.

Now if $\mathscr{E}$ is an allowable extension in $\mathbb{A}$ !-bimod then the last lemma implies that $\overline{\mathscr{E}} e$ is an allowable extension in $\left(\langle\mathscr{J}\rangle^{*} \mathbb{A}\right)$ !-bimod. Further, if $\mathscr{E} \leftarrow \mathscr{F} \rightarrow \mathscr{E}$ is an equivalence of extensions in $\mathbb{A}$ !-bimod then $\overline{\mathscr{E}}_{e} \leftarrow \overline{\mathscr{F}} e \rightarrow \overline{\mathscr{E}^{\prime}} e$ is also an equivalence. So, for each $\mathbb{A}$ !-bimodule $\mathfrak{b}$ and $\left(\langle\mathscr{J}\rangle^{*} \mathbb{A}\right)$ !-bimodule $\mathfrak{c},(3.1)$ extends to a map

$$
\operatorname{Ext}_{\mathbb{A} !-\mathbb{A} !}^{\bullet}(\mathfrak{b}, \mathfrak{c}) \rightarrow \operatorname{Ext}_{\left(\langle\mathscr{J}\rangle^{*} \mathbb{A}\right) !-\left(\langle\mathscr{J}\rangle^{*} \mathbb{A}\right) !}(\overline{\mathfrak{b}} e, \mathfrak{c}):[\mathscr{E}] \mapsto[\overline{\mathscr{E}} e] .
$$

While (3.1) is a monomorphism, in general the latter map is not. For an example, let $\mathscr{I}=\{0<1\}$ and $\mathscr{J}=\{1\}$, so that $e=\varphi^{11}$. Then

$$
\mathscr{E}: 0 \rightarrow \overline{\mathbb{A} !} \otimes(1-e) \mathbb{A} ! e \rightarrow \overline{\mathbb{A} !} \otimes(1-e) \mathbb{A} ! \rightarrow \overline{\mathbb{A} !} \otimes(\mathbb{A} ! / \mathbb{A} ! e) \rightarrow 0
$$

is a nontrivial allowable extension in $\mathbb{A} !$-bimod while $[\overline{\mathscr{E}} e]=0$ since $(\overline{\mathbb{A} !} \otimes(\mathbb{A} ! / \mathbb{A} ! e)) e$ $=0$. In contrast we have the following lemma. 
LEMMA 3.5. Let $\mathfrak{b}$ be an $\mathbb{A}$ !-bimodule and $\mathfrak{c}$ be $a\left(\langle\mathscr{J}\rangle^{*} \mathbb{A}\right)$ !-bimodule. Then $\operatorname{Ext}_{\mathbb{A} !-\mathbb{A} !}^{\bullet}(\mathfrak{b}, \mathfrak{c}) \rightarrow \operatorname{Ext}_{\left(\langle\mathcal{J}\rangle^{*} \mathbb{A}\right) !-\left(\langle\mathcal{J}\rangle^{*} \mathbb{A}\right) !}(\overline{\mathfrak{b}} e, \mathfrak{c})$ is an isomorphism if $\overline{\mathfrak{b}}=\overline{\mathfrak{b}} e$.

ProOF. Let $\pi: \mathfrak{b} \rightarrow \overline{\mathfrak{b}}=\overline{\mathfrak{b}} e$ be the projection. We shall show that

$$
\operatorname{Ext}_{\left(\langle\mathscr{J}\rangle^{*} \mathrm{~A}\right) !-\left(\langle\mathscr{J}\rangle^{*} \mathbf{A}\right) !}(\overline{\mathfrak{b}}, \mathfrak{c}) \rightarrow \operatorname{Ext}_{\mathrm{A} !-\mathrm{A} !}^{\bullet}(\mathfrak{b}, \mathfrak{c}):[\mathscr{F}] \rightarrow[\mathscr{F} \pi]
$$

is the inverse to $[\mathscr{E}] \mapsto[\overline{\mathscr{E}} e]$. The comments following $(3.1)$ make this obvious in dimension zero. For the case of dimension $n>0$ first suppose that $[\mathscr{F}] \in$ $\operatorname{Ext}_{\left(\langle\mathcal{J}\rangle^{*} \mathbb{A}\right) !-(\langle\mathcal{J}\rangle * \mathbb{A}) !}^{n}(\overline{\mathfrak{b}}, \mathfrak{c})$. Since $(1-e) \mathfrak{d}=0$ and $\mathfrak{d} e=\mathfrak{d}$ for any $\left(\langle\mathscr{J}\rangle^{*} \mathbb{A}\right) !$-bimodule

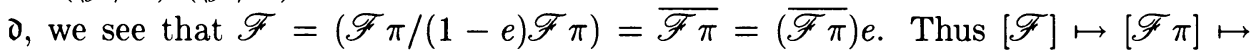
$[(\overline{\mathscr{F} \pi}) e]$ is the identity. Now suppose that $[\mathscr{E}] \in \operatorname{Ext}_{\mathrm{A} !-\mathrm{A} !}^{n}(\mathfrak{b}, \mathfrak{c})$. From Theorem $3.3(3)$ we see that $(1-e) \mathscr{E}$ is an $\mathbb{A}$ !-bimodule extension. Since $(1-e) \mathfrak{c}=0$, the inclusion $(1-e) \mathscr{E} \mapsto \mathscr{E}$ yields a quotient map $\mathscr{E} \rightarrow \overline{\mathscr{E}}$ which starts with $\operatorname{Id}_{\mathfrak{c}}: \mathfrak{c} \rightarrow \mathfrak{c}$ and ends with $\pi$. Hence there is a congruence of $\mathbb{A}$ !-bimodule extensions, $\mathscr{E}=\operatorname{Id}_{c} \mathscr{E} \equiv \overline{\mathscr{E}}_{\pi}$. Using the evident forgetful functor we can view $\overline{\mathscr{E}} e$ as an $\mathbb{A} !-$ bimodule extension. Then the remarks following Theorem 3.3 show that there is a natural inclusion $\overline{\mathscr{E}} e \nrightarrow \overline{\mathscr{E}}$. But, since $\mathfrak{c}=\overline{\mathfrak{c}}=\overline{\mathfrak{c}} e$ and $\overline{\mathfrak{b}}=\overline{\mathfrak{b}} e$, that inclusion is an equivalence. Hence, $\mathscr{E} \equiv \overline{\mathscr{E}}_{\pi} \equiv\left(\overline{\mathscr{E}}_{e}\right) \pi$ and $[\mathscr{E}] \mapsto\left[\overline{\mathscr{E}}_{e}\right] \mapsto\left[\left(\overline{\mathscr{E}}_{e}\right) \pi\right]$ is the identity.

Note that the inverse for $[\mathscr{E}] \mapsto[\overline{\mathscr{E}} e]$ supplied in the proof above is not defined if $\overline{\mathfrak{b}} e \neq \overline{\mathfrak{b}}$. Next, we examine $\overline{\mathbb{N}}$ !.

LEMMA 3.6. If $\mathbb{N}$ is an $\mathbb{A}$-bimodule then there is an $\mathbb{A}$ !-bimodule isomorphism $\overline{\mathbb{N} !} \rightarrow\left(\langle\mathscr{J}\rangle^{*} \mathbb{N}\right) !$. Further, $\overline{\mathbb{N}} ! e=\overline{\mathbb{N} !}$ and the exact sequence $0 \rightarrow(1-e) \mathbb{N} ! \rightarrow \mathbb{N} ! \rightarrow$ $\left(\langle\mathscr{J}\rangle^{*} \mathbb{N}\right) ! \rightarrow 0$ is allowable.

PROOF. The first assertion immediately implies the others. Now, using the definitions of $\mathbb{N}$ ! and the idempotent $e$ we see that $(1-e) \mathbb{N} !=\prod_{i \notin \mathcal{J}} \bigsqcup_{j \geq i} \mathbb{N}^{i} \varphi^{i j}$. Thus, there is an obvious $k$-bimodule isomorphism $\overline{\mathbb{N} !}=\mathbb{N} ! /(1-e) \mathbb{N} ! \cong \prod_{i \in \mathcal{J}} \coprod_{j \geq i} \mathbb{N}^{i} \varphi^{i j}$ $=\left(\langle\mathscr{J}\rangle^{*} \mathbb{N}\right)$ !, which is easily seen to be an $\mathbb{A}$ !-bimodule map.

All is now in place to prove a version of Theorem 3.2 for diagram rings.

THEOREM 3.7. If $\mathbb{N}$ is an $\mathbb{A}$-bimodule and $\mathbb{M}$ is $a\langle\mathscr{J}\rangle^{*} \mathbb{A}$-bimodule then

$$
\operatorname{Ext}_{\mathbb{A} !-\mathbb{A} !}^{\bullet}\left(\mathbb{N} !,\left(\langle\mathscr{J}\rangle_{*} \mathbb{M}\right) !\right) \cong \operatorname{Ext}_{\left(\langle\mathscr{J}\rangle^{*} \mathbb{A}\right) !-\left(\langle\mathscr{J}\rangle^{*} \mathbb{A}\right) !}\left(\left(\langle\mathscr{J}\rangle^{*} \mathbb{N}\right) !, \mathbb{M} !\right)
$$

ProOF. As usual, we may view $\mathbb{M}$ ! as an $\mathbb{A}$ !-bimodule through $\mathbb{A} ! \rightarrow\left(\langle\mathscr{J}\rangle^{*} \mathbb{A}\right)$ !. Since $\left(\langle\mathscr{J}\rangle_{*} \mathbb{M}\right)^{i}=0$ for $i \notin \mathscr{J}$ it follows that $\varphi^{h h}\left(\langle\mathscr{J}\rangle_{*} \mathbb{M}\right) ! \varphi^{i i}=0$ if either $h \notin \mathscr{J}$ or $i \notin \mathscr{J}$. This implies that $\left(\langle\mathscr{J}\rangle_{*} \mathbb{M}\right)$ ! and $\mathbb{M}$ ! are isomorphic as $\mathbb{A}$ !-bimodules. In view of the last lemma, the theorem is merely an instance of Lemma 3.5.

We shall call a poset $\mathscr{I}$ a cone if it has a least element 0 . So $0 \leq i$ for every $i \in \mathscr{I}$. The cone is trivial if it consists of 0 alone. A principal filter in an arbitrary poset is a cone. Such a cone is trivial if and only if the generating element is maximal in the poset. Granting the CCT for a moment, Theorem 3.2 informs us that Theorem 3.7 must hold with $\mathscr{J}$ replaced by $i$. This, for the case in which $\mathscr{J}$ is a cone and $i=0$, is what we prove in $\S 4$. It immediately yields the next theorem whose relevance to the CCT is detailed in Theorem 3.8 . 
THE SECOND ACYCLICITY THEOREM. Let $\mathscr{I}$ be a cone with least element 0 and $\mathbb{A}$ be a diagram over $\mathscr{I}$. If $\mathbb{E}$ and $\mathbb{N}$ are $\mathbb{A}$-bimodules and $\mathbb{E}$ is a 0 -primitive relative injective then $\mathbb{E}$ ! is a relative $\operatorname{Hom}_{\mathbb{A} !-\mathbb{A} !}(\mathbb{N} !,-)$-acyclic bimodule; that is, $\operatorname{Ext}_{\mathbf{A} !-\mathbf{A} !}^{n}(\mathbb{N} !, \mathbb{E} !)=0$ for $n>0$.

The results of this section have been designed to prove

\section{THEOREM 3.8. The CCT is equivalent to the Second Acyclicity Theorem.}

PrOOF. Clearly, we need only show that the Second Acyclicity Theorem implies the first. For that, let $\mathscr{I}$ be a poset, $\mathbb{A}$ be a diagram over $\mathscr{I}$, and $\mathbb{N}$ be an $\mathbb{A}$-bimodule. Choose an arbitrary element $0 \in \mathscr{I}$, let $\mathscr{J}=0 / \mathscr{I}$ be the principal filter generated by 0 , and let $\mathbb{E}$ be a 0 -primitive relative injective $\mathbb{A}$-bimodule. Then $\langle\mathscr{J}\rangle^{*} \mathbb{E}$ is the 0 -primitive relative injective $\left(\langle\mathscr{J}\rangle^{*} \mathbb{A}\right)$-bimodule determined by the $\mathbb{A}^{0}$-bimodule $\mathbb{E}^{0}$. Moreover, $\mathbb{E}=\langle\mathscr{J}\rangle_{*}\left(\langle\mathscr{J}\rangle^{*} \mathbb{E}\right)$. Applying the Second Acyclicity Theorem and Theorem 3.7 to $\mathscr{J},\langle\mathscr{J}\rangle^{*} \mathbb{A},\langle\mathscr{J}\rangle^{*} \mathbb{N}$ and $\langle\mathscr{J}\rangle^{*} \mathbb{E}$ then yields

$$
\begin{aligned}
\operatorname{Ext}_{\mathrm{A} !-\mathrm{A} !}^{n}(\mathbb{N} !, \mathbb{E} !) & =\operatorname{Ext}_{\mathrm{A} !-\mathrm{A} !}^{n}\left(\mathbb{N} !,\left(\langle\mathscr{J}\rangle_{*}\langle\mathscr{J}\rangle^{*} \mathbb{E}\right) !\right) \\
& =\operatorname{Ext}_{\left(\langle\mathscr{J}\rangle^{*} \mathbb{A}\right) !-\left(\langle\mathscr{J}\rangle^{*} \mathrm{~A}\right) !}^{n}\left(\left(\langle\mathcal{J}\rangle^{*} \mathbb{N}\right) !,\left(\langle\mathscr{J}\rangle^{*} \mathbb{E}\right) !\right)=0
\end{aligned}
$$

for $n>0$. That is, $\mathbb{E}$ ! is a relative $\operatorname{Hom}_{\mathbb{A} !-\mathbb{A} !}(\mathbb{N} !,-)$-acyclic bimodule and we are done.

We should remark that neither Theorem 3.8 nor the Second Acyclicity Theorem appear in [GS3], where we consider the special case $\mathscr{J}=\{0<1\}$ of the CCT. In that case the Acyclicity Theorem requires that we examine $i$-primitive relative injectives for $i=0$ and $i=1$. The proof above then permits us to reduce the poset to $i / \mathcal{F}$. Now for $i=0$ this affords no reduction at all. So these theorems could only be useful in the case $i=1$. However, it is not hard to show that $\mathbb{E}$ ! is a relative injective $\mathbb{A}$ !-bimodule for every 1-primitive relative injective $\mathbb{E}$. (This is equivalent to the maximality of 1 in $\mathscr{J}$, as we prove in $\S 5$.)

In the context of arbitrary diagrams we note one further result which will play crucial roles in $\S 4$ and $\S 6$. Suppose that $\mathscr{I}$ is a set (which we do not assume to be partially ordered) and that $A$ is a $k$-algebra containing $R=\prod_{\mathscr{J}} k=k^{\mathcal{J}}$ as a subalgebra. There is then a set of pairwise orthogonal idempotents $\left\{e^{i}\right\}_{i \in \mathcal{I}}$ in $A$ whose (possibly infinite) sum is the unit of $A$. Observe that the $k$-bimodule $A^{\prime}=\prod_{i \in \mathcal{I}} \coprod_{j \in \mathscr{I}} e^{i} A e^{j}$ is, in a natural way, a $k$-algebra containing $R$. We say that $A$ is an $\mathscr{I}$-aligned algebra if the natural map $A \rightarrow \prod_{i} \prod_{j} e^{i} A e^{j}: a \mapsto\left(e^{i} a e^{j}\right)$, establishes a $k$-algebra isomorphism $A \cong A^{\prime}$. Now let $\Omega \subset \mathscr{I} \times \mathscr{I}$ be an arbitrary binary relation on $\mathscr{J}$. The examples of greatest interest to us will be $\Omega_{\infty}=\mathscr{I} \times \mathscr{J}$ and, when $\mathscr{F}$ is a poset, $\Omega_{\leq}=\{(i, j) \mid i \leq j\}$. Then, for any $A$-bimodule $M$ the $k$-bimodule $M_{\Omega}=\prod_{i \in \mathcal{I}} \coprod_{(i, j) \in \Omega} e^{i} M e^{j}$ is an $A^{\prime}$-bimodule in an obvious way. Further, if $f \in \operatorname{Hom}_{A-A}(N, M)$ then $f\left(e^{i} N e^{j}\right) \subset e^{i} M e^{j}$ and $f_{\Omega}=\left.\prod \amalg f\right|_{e^{i} N e^{j}}$ is an $A^{\prime}$-bimodule map $N_{\Omega} \rightarrow M_{\Omega}$. Clearly then, $M \leadsto M_{\Omega}$ is a functor from $A$-bimod to $A^{\prime}$-bimod. We henceforth assume that $A$ is aligned and identify $A$ with $A^{\prime}$. By definition, $M$ will be an $\Omega$-aligned $A$-bimodule if and only if $M=M_{\Omega}$, i.e. the canonical map $M \rightarrow \prod_{i} \prod_{j} e^{i} M e^{j}: m \mapsto\left(e^{i} m e^{j}\right)$ is an isomorphism $M \cong$ $\prod_{i \in \mathscr{I}} \coprod_{(i, j) \in \Omega} e^{i} M e^{j}=M_{\Omega}$. In particular, for each $i \in \mathscr{J}$ and $m \in M$ the set of $j \in \mathscr{I}$ for which $e^{i} m e^{j} \neq 0$ is finite; that is, $e^{i} m e^{j}=0$ for almost all $j \in \mathscr{J}$. (We could, of course, have defined $\Omega$-alignment for algebras. This would have gained us 
nothing, however, since $A$ is $\Omega$-aligned if and only if it is $\mathscr{F}$-aligned and $\Omega$ contains $\left\{(i, j) \in \mathscr{I} \times \mathscr{I} \mid e^{i} A e^{j} \neq 0\right\}$.) An $\Omega$-aligned map, $f: N \rightarrow M$, between $\Omega$-aligned bimodules is one for which $f\left(\sum n^{i}\right)=\sum f\left(n^{i}\right)$ whenever $n^{i} \in e^{i} N$. For any map $f \in \operatorname{Hom}_{A-A}(N, M)$ the induced map $f_{\Omega} \in \operatorname{Hom}_{A-A}\left(N_{\Omega}, M_{\Omega}\right)$ is $\Omega$-aligned. In fact, since $M \rightsquigarrow M_{\Omega}$ is idempotent-i.e. $\left(M_{\Omega}\right)_{\Omega}=M_{\Omega}$-we see that its image is precisely the subcategory of $\Omega$-aligned bimodules and maps. Observe that if $\mathscr{I}$ is finite then $A$ is automatically aligned and all $A$-bimodules and maps are $\Omega_{\infty}$ aligned. We shall call $M$ an $\Omega$-hostile A-bimodule if $M=\prod_{i \in \mathcal{I}} e^{i} M$ but $e^{i} M e^{j}=0$ whenever $(i, j) \in \Omega$.

Naturally, we define an $\Omega$-aligned extension to be one in which all bimodules and maps are $\Omega$-aligned. Trivially, $M \leadsto M_{\Omega}$ is exact and preserves allowability. Hence, it induces a natural transformation $\operatorname{Ext}_{A-A}^{\circ}(-,-) \rightarrow \operatorname{Ext}_{A-A}^{\bullet}(-\Omega,-\Omega):\left[\mathscr{E}^{\circ} \rightsquigarrow\left[\mathscr{E}_{\Omega}\right]\right.$. It is immediate that an extension $\mathscr{E}$ is $\Omega$-aligned if and only if $\mathscr{E}=\mathscr{E}_{\Omega}$.

THEOREM 3.9. Let $A$ and $N$ be, respectively, an $\mathscr{I}$-aligned algebra and an $\Omega$-aligned A-bimodule.

(1) If $M$ is an $\Omega$-hostile $A$-bimodule then $\operatorname{Ext}_{A-A}^{\bullet}(N, M)=0$.

(2) If $M$ is an $\Omega$-aligned A-bimodule then $[\mathscr{E}] \rightsquigarrow\left[\mathscr{E}_{\Omega}\right]$ is the identity map $\operatorname{Ext}_{A-A}^{n}(N, M) \rightarrow \operatorname{Ext}_{A-A}^{n}(N, M)$ for $n>0$. That is, for $n>0$ every class $[\mathscr{E}] \in \operatorname{Ext}_{A-A}^{n}(N, M)$ can be represented by an $\Omega$-aligned extension, namely $\mathscr{E} \equiv \mathscr{E}_{\Omega}$.

Proof. If $L$ is an $A$-bimodule then $L^{\dagger}=\prod_{i \in \mathscr{I}} e^{i} L$ is an $A$-bimodule in a natural way and, as above, $L \leadsto L^{\dagger}$ is an exact functor which preserves allowability. There are obvious maps $L \rightarrow L^{\dagger}$ and $L_{\Omega} \rightarrow L^{\dagger}$, which are natural in $L$. Clearly, $N=N^{\dagger}=N_{\Omega}$ and, whether hostile or aligned, $M=M^{\dagger}$. Now suppose that $n>0$ and that $[\mathscr{E}] \in \operatorname{Ext}_{A-A}^{n}(N, M)$ is arbitrary. Then $\mathscr{E} \rightarrow \mathscr{E}^{\dagger}$ starts and ends with identity maps-so $\mathscr{E} \equiv \mathscr{E}^{\dagger}$-while $\mathscr{E}_{\Omega} \rightarrow \mathscr{E}^{\dagger}$ has an identity map as its end. When $M$ is aligned $\mathscr{E}_{\Omega} \rightarrow \mathscr{E}^{\dagger}$ also starts with an identity map, so $\mathscr{E} \equiv \mathscr{E}^{\dagger} \equiv \mathscr{E}_{\Omega}$ and we have proved half of (2). The other half follows since $\mathscr{E}_{\Omega}$ is then an aligned representative for [ $\mathscr{E}]$. On the other hand, if $M$ is hostile then $M_{\Omega}=0$ and, so, the map $\mathscr{E}_{\Omega} \rightarrow \mathscr{E}^{\dagger}$ starts with $0 \rightarrow M$. Hence, $0=0 \mathscr{E}_{\Omega} \equiv \mathscr{E}^{\dagger} \mathrm{Id}=\mathscr{E}^{\dagger}$ and $\mathscr{E} \equiv 0$, which establishes (1) in dimension $n>0$. Finally, for $g \in \operatorname{Hom}_{A-A}(N, M)=$ $\operatorname{Ext}_{A-A}^{0}(N, M)$ and $(i, j) \in \Omega$ we have $g\left(e^{i} N e^{j}\right)=e^{i} g(N) e^{j} \subset e^{i} M e^{j}=0$. Hence $g(N)=0$ and $\operatorname{Ext}_{A-A}^{0}(N, M)=0$.

If $\mathbb{A}$ is a diagram over a poset $\mathscr{I}$ then $\mathbb{A}$ ! is an $\mathscr{I}$-aligned algebra. (The idempotent $e^{i}$ is, of course, just $\varphi^{i i}$.) Likewise, if $\mathbb{M}$ is an $\mathbb{A}$-bimodule then $\mathbb{M}$ ! is an $\Omega_{\leq}$-aligned $\mathbb{A}$ !-bimodule. In fact, the $\mathbb{A}$ !-bimodules in the image of !: $\mathbb{A}$-bimod $\rightarrow \mathbb{A}$ !-bimod can be characterized intrinsically (up to isomorphism). Specifically, an $\mathbb{A}$ !-bimodule $\mathfrak{c}$ is isomorphic to $\mathbb{C}$ ! for some $\mathbb{A}$-bimodule $\mathbb{C}$ if and

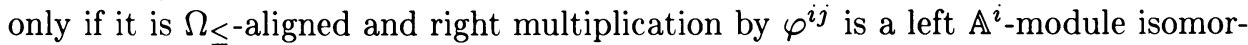
phism - $\cdot \varphi^{i j}: \varphi^{i i} \mathfrak{c} \varphi^{i i} \rightarrow \varphi^{i i} \mathfrak{c} \varphi^{j j}$ for all $i \leq j$. (To construct the $\mathbb{A}$-bimodule $\mathbb{C}$, set $\mathbb{C}^{i}=\varphi^{i i} \mathfrak{c} \varphi^{i i}$; the map $T^{i j}: \mathbb{C}^{j} \rightarrow \mathbb{C}^{i}$ is then uniquely determined by the equation $T^{i j}(x) \varphi^{i j}=\varphi^{i j} x$.) A (reasonable) functorial construction of an $\mathbb{A}$ !-bimodule with both of these properties from an $\Omega_{\leq}$-aligned bimodule would, in view of the theorem above, yield a proof of the CCT. We have been unable to produce such a construction.

We conclude this section with a correction to (and generalization of) the final exercise left at the end of [GS1, §16]. Suppose that $\mathscr{I}$ is a cone in which 
$\{i>0\}$ is also a cone, that $\mathbb{M}$ and $\mathbb{N}$ are $\mathbb{A}$-bimodules, and that $\mathbb{M}^{i}=0$ for $i \neq 0$ while $\mathbb{N}^{0}=0$. Denote the least element of $\{i>0\}$ by 1 and set $A=\mathbb{A}^{1}$. We claim that $\operatorname{Ext}_{\mathbb{A}-\mathbb{A}}^{\bullet}(\mathbb{N}, \mathbb{M}) \cong \operatorname{Ext}_{A-A}^{\bullet-1}\left(\mathbb{N}^{1}, \mathbb{M}^{0}\right)$. To prove this, apply $\operatorname{Ext}_{\mathbb{A}-\mathbb{A}}^{\bullet}(\mathbb{N},-)$ to $\mathscr{E}: 0 \rightarrow \mathbb{M} \rightarrow\langle 0\rangle_{*} \mathbb{M}^{0} \rightarrow\langle 1\rangle_{*} \mathbb{M}^{0} \rightarrow 0$. Since $\mathbb{N}^{0}=0$, Theorem 3.2 implies that $\operatorname{Ext}_{\mathbb{A}-\mathbb{A}}^{\bullet}\left(\mathbb{N},\langle 0\rangle_{*} \mathbb{M}^{0}\right)=0$. It then follows that $\operatorname{Ext}_{\mathbb{A}-\mathbb{A}}^{\bullet}(\mathbb{N}, \mathbb{M}) \cong \operatorname{Ext}_{\mathbb{A}-\mathbb{A}}^{\bullet-1}\left(\mathbb{N},\langle 1\rangle_{*} \mathbb{M}^{0}\right)$ and applying Theorem 3.2 once more finishes the proof.

4. Proof of the Second Acyclicity Theorem. This section is devoted to the proof of the Second Acyclicity Theorem. Consequently, we assume throughout that $\mathscr{I}$ is a cone with least element 0 . We denote the filter $\{i>0\}$ by $\mathscr{J}$ and set $e=$ $\sum_{\mathscr{J}} \varphi^{j j}$, the idempotent determined by $\mathscr{J}$. So $1-e=\varphi^{00}$. Theorem 3.3 and the comments following it then apply. In particular, $\varphi^{00} \mathbb{A}$ ! and $\mathbb{A}$ !e are two-sided ideals in $\mathbb{A}$ ! and there is a $k$-algebra epimorphism $\mathbb{A} ! \rightarrow\left(\langle\mathscr{J}\rangle^{*} \mathbb{A}\right)$ ! whose kernel is $\varphi^{00} \mathbb{A}$ !. The obvious $k$-module isomorphism $\mathbb{A} ! / \mathbb{A} ! e \rightarrow \mathbb{A} !(1-e)=\mathbb{A} ! \varphi^{00}=\mathbb{A}^{0} \varphi^{00} \cong \mathbb{A}^{0}$ is, similarly, an algebra map and the forgetful functors induced by $\mathbb{A} ! \rightarrow\left(\langle\mathscr{J}\rangle^{*} \mathbb{A}\right)$ ! and $\mathbb{A} ! \rightarrow \mathbb{A}^{0}$ are exact and preserve allowability. In addition, since $\varphi^{00} \mathbb{A}$ ! is projective as a right $\mathbb{A}$ !-module, $\varphi^{00} \mathbb{A} ! \otimes_{\mathbb{A} !}-: \mathbb{A} !$-bimod $\rightarrow\left(\mathbb{A}^{0}-\mathbb{A} !\right)$-bimod is likewise an exact functor which preserves allowability. In fact, it is naturally equivalent to $\mathfrak{a} \rightsquigarrow \varphi^{00} \mathfrak{a}$; the isomorphism $\varphi^{00} \mathbb{A} ! \otimes_{\mathbb{A} !} \mathfrak{a} \rightarrow \varphi^{00} \mathfrak{a}$ is simply $r \otimes a \mapsto r a$ and we shall use it to identify these two modules whenever convenient. Of course, $\varphi^{00} \mathbb{A} ! \otimes_{\mathbb{A} !}-$ is right adjoint to the evident forgetful functor and using the latter functor we may view $\varphi^{00} \mathfrak{a}=\varphi^{00} \mathbb{A}$ ! $\otimes_{\mathbb{A} !} \mathfrak{a}$ as an $\mathbb{A}$ !-bimodule. The structure it so acquires is then identical with its natural structure as an $\mathbb{A}$ !-subbimodule of $\mathfrak{a}$. (That is, the counit of the adjunction is the identity.) Likewise, as noted after Theorem 3.3, the two possible $\mathbb{A}$ !-bimodule structures on $\mathfrak{a} e$ coincide. Furthermore, if $\mathbb{M}$ is an $\mathbb{A}$-bimodule and $\mathbb{M}^{0}$ is viewed as an $\mathbb{A} !$-bimodule through $\mathbb{A} ! \rightarrow \mathbb{A}^{0}$, then the obvious $k$-module isomorphism $\mathbb{M} ! / \mathbb{M} ! e \rightarrow \mathbb{M} !(1-e)=\mathbb{M}^{0} \varphi^{00} \cong \mathbb{M}^{0}$ is an $\mathbb{A} !$-bimodule map and $0 \rightarrow \mathbb{M} ! e \rightarrow \mathbb{M} ! \rightarrow \mathbb{M}^{0} \rightarrow 0$ is an allowable short exact sequence.

Now let $M$ be an $\mathbb{A}^{0}$-bimodule. For the remainder of this section we set $\mathbb{M}=$ $\langle 0\rangle_{*} M$ and $\mathfrak{m}=\mathbb{M} ! e$. So, each $\mathbb{M}^{i}=M$, every $\mathbb{M}^{j} \rightarrow \mathbb{M}^{i}=\operatorname{Id}_{M}$, and the allowable exact sequence above becomes

$$
0 \rightarrow \mathfrak{m} \rightarrow \mathbb{M} ! \rightarrow M \rightarrow 0 .
$$

Our goal is to prove that $\operatorname{Ext}_{\mathbb{A}^{!}-\mathbb{A}^{!} !}^{\bullet}(\mathbb{N} !, \mathbb{M} !) \cong \operatorname{Ext}_{\mathbb{A}^{0}-\mathbb{A}^{0}}^{\circ}\left(\mathbb{N}^{0}, M\right)$ for every $\mathbb{A}$-bimodule $\mathbb{N}$. The Second Acyclicity Theorem will follow from this immediately. In view of (4.1) it suffices to prove that $\operatorname{Ext}_{\mathbb{A} !-\mathrm{A} !}^{\bullet}(\mathbb{N} !, \mathfrak{m})=0$ and $\operatorname{Ext}_{\mathbf{A} !-\mathrm{A} !}^{\bullet}(\mathbb{N} !, M) \cong$ $\operatorname{Ext}_{\mathrm{A}^{0}-\mathbb{A}^{0}}^{\circ}\left(\mathbb{N}^{0}, M\right)$. The second of these is a consequence of the following two lemmas.

LEMMA 4.1. If $N$ is an $\mathbb{A}^{0}$-bimodule then $\operatorname{Ext}_{\mathbb{A}^{0}-\mathbb{A}^{0}}^{\circ}(N, M) \rightarrow \operatorname{Ext}_{\mathbb{A}^{!}-\mathbb{A}^{!}}^{\bullet}(N, M)$ : $[\mathscr{E}] \mapsto[\mathscr{E}]$ is an isomorphism.

Proof. The assertion of the lemma is trivial in dimension zero. Now suppose that $n>0$ and $[\mathscr{E}] \in \operatorname{Ext}_{\mathrm{A} ! \mathrm{A} !}^{\bullet}(N, M)$. Then there are inclusions of allowable

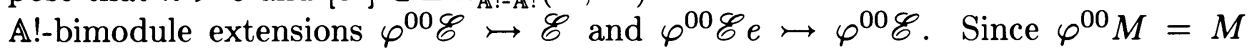
and $\varphi^{00} N=N$, the first of these is a congruence: $\varphi^{00 \mathscr{E}} \equiv \mathscr{E}$. Also, since $\varphi^{00 \mathscr{E}}=\varphi^{00} \mathscr{E} e+\varphi^{00} \mathscr{E} \varphi^{00}$, the second of these has an exact quotient, $\varphi^{00} \mathscr{E} \varphi^{00}$, and $\varphi^{00} \mathscr{E} \rightarrow \varphi^{00} \mathscr{E} \varphi^{00}$ is again a congruence. Now the modules in $\varphi^{00} \mathscr{E} \varphi^{00}$ all have the form $\varphi^{00} \mathfrak{a} \varphi^{00}$ and, so, are naturally $\mathbb{A}^{0}$-bimodules. The correspon- 
dence $\mathscr{E} \mapsto \varphi^{00} \mathscr{E} \varphi^{00}$ is easily seen to respect equivalence. The congruence $\mathscr{E} \equiv$ $\varphi^{00} \mathscr{E} \varphi^{00}$ in $\mathbb{A} !$-bimod then shows that $[\mathscr{E}] \mapsto\left[\varphi^{00} \mathscr{E} \varphi^{00}\right]$ provides an inverse to $\operatorname{Ext}_{\mathrm{A}^{0}-\mathbb{A}^{0}}^{0}(N, M) \rightarrow \operatorname{Ext}_{\mathbb{A}^{\prime} ! \mathrm{A} !}^{\bullet}(N, M)$.

LEMMA 4.2. Let $\mathbb{N}$ be an $\mathbb{A}$-bimodule. Then $\operatorname{Ext}_{\mathbb{A} !-\mathbb{A} !}^{\bullet}(\mathbb{N} ! e, M)=0$ and there is an isomorphism $\operatorname{Ext}_{\mathrm{A} !-\mathrm{A} !}^{\cdot}(\mathbb{N} !, M) \cong \operatorname{Ext}_{\mathbb{A} !-\mathbb{A} !}^{\cdot}\left(\mathbb{N}^{0}, M\right)$.

PROOF. The second claim follows immediately from the first by examining the long exact sequence induced by the allowable exact sequence $0 \rightarrow \mathbb{N} ! e \rightarrow$ $\mathbb{N} ! \rightarrow \mathbb{N}^{0} \rightarrow 0$. For the dimension zero case of the first claim, suppose that $f \in \operatorname{Hom}_{\mathrm{A} !-\mathrm{A} !}(\mathbb{N} ! e, M)$. Then, since $e$ is an idempotent we see that $f(\mathbb{N} ! e) \subset M e=0$ and, so, $f=0$. Now let $\mathscr{E}$ represent a class in $\operatorname{Ext}_{\mathrm{A} !-\mathrm{A} !}^{n}(\mathbb{N} ! e, M)$ for some $n \neq 0$. The map of extensions $\mathscr{E} e \rightarrow \mathscr{E}$ starts with $0 \rightarrow M$ and ends with an identity map. Thus, $0=0(\mathscr{E} e) \equiv \mathscr{E}$ Id $=\mathscr{E}$ and $[\mathscr{E}]=0$.

COROLlaRY 4.3. Let $\mathbb{N}$ be an $\mathbb{A}$-bimodule. Then there is an isomorphism $\operatorname{Ext}_{\mathbb{A}^{!}-\mathbb{A}^{!} !}^{\bullet}(\mathbb{N} !, M) \cong \operatorname{Ext}_{\mathrm{A}^{0}-\mathbb{A}^{0}}^{\circ}\left(\mathbb{N}^{0}, M\right)$.

Our proof that $\operatorname{Ext}_{\mathbf{A} !-\mathrm{A} !}^{\bullet}(-!, \mathfrak{m})=0$ requires the right adjoint to $\varphi^{00} \mathbb{A} ! \otimes_{\mathbb{A} !}$ $-: \mathbb{A} !$-bimod $\rightarrow\left(\mathbb{A}^{0}\right.$ - $\left.\mathbb{A} !\right)$-bimod, namely $\operatorname{Hom}_{\mathbb{A}^{0}}\left(\varphi^{00} \mathbb{A} !,-\right)$, and the unit of the adjunction. For any $\left(\mathbb{A}^{0}-\mathbb{A}\right.$ !)-bimodule $\mathscr{M}$ the $\mathbb{A}$ !-bimodule structure of $\operatorname{Hom}_{\mathbb{A}^{0}}\left(\varphi^{00} \mathbb{A} !, \mathscr{M}\right)$ is, of course, derived from the right $\mathbb{A} !$-module structures of $\varphi^{00} \mathbb{A}$ ! and $\mathscr{M}$ : for $r, s \in \mathbb{A}$ ! and $f \in \operatorname{Hom}_{\mathbb{A}^{0}}\left(\varphi^{00} \mathbb{A} !, \mathscr{M}\right)$ the left $\mathbb{A}^{0}$-module map $r f s$ is defined by $(r f s)(x)=f(x r) s$. In particular, since $\varphi^{00} \mathbb{A} !=\amalg \mathbb{A}^{0} \varphi^{0 i}$, we have

$$
\varphi^{i i} \operatorname{Hom}_{\mathbb{A}^{0}}\left(\varphi^{00} \mathbb{A} !,-\right)=\operatorname{Hom}_{\mathbb{A}^{0}}\left(\mathbb{A}^{0} \varphi^{0 i},-\right) .
$$

Now when $i=0$ the left-hand side of the latter equation is simply a composite of the two adjoints while, since the $\mathbb{A}^{0}$-bimodule $\mathbb{A}^{0} \varphi^{00}$ is free as a left $\mathbb{A}^{0}$-module, the right-hand side is naturally equivalent to the identity functor. (Put another way, the counit of the adjunction is an isomorphism.) The other composite, which we denote more succinctly by $\mathbb{A} !$-bimod $\rightarrow \mathbb{A}$ !-bimod: $\mathfrak{a} \leadsto \tilde{\mathfrak{a}}$, is then idempotent (up to natural equivalence); i.e., $\widetilde{\mathfrak{a}}=\operatorname{Hom}_{\mathbb{A}^{0}}\left(\varphi^{00} \mathbb{A} !, \varphi^{00} \mathfrak{a}\right)$ and the unit of the adjunction (which will be described explicitly below) is a natural isomorphism $\eta_{\tilde{a}}: \widetilde{\mathfrak{a}} \rightarrow(\mathfrak{a})^{\sim}$. Since the left $\mathbb{A}^{0}$-module $\varphi^{00} \mathbb{A} !=\amalg \mathbb{A}^{0} \varphi^{0 i}$ is free, $\operatorname{Hom}_{\mathbb{A}^{0}}\left(\varphi^{00} \mathbb{A} !,-\right)$ is exact and preserves allowability. Hence $\mathfrak{a} \rightsquigarrow \widetilde{\mathfrak{a}}$, being a composite of functors with these properties, is likewise an exact functor which preserves allowability. Moreover, applying the standard isomorphism $\operatorname{Hom}_{\mathbb{A}^{0}}\left(\varphi^{00} \mathbb{A} !,-\right) \cong \prod_{\mathscr{Y}} \operatorname{Hom}_{\mathbb{A}^{0}}\left(\mathbb{A}^{0} \varphi^{0 i},-\right)$ and $(4.2)$ to $\varphi^{00} \mathfrak{a}$ yields

$$
\tilde{\mathfrak{a}}=\prod \varphi^{i i} \tilde{\mathfrak{a}}
$$

and we may safely write $a=\sum \varphi^{i i} a$ for $a \in \tilde{\mathfrak{a}}$.

The unit of the adjunction is the natural transformation which assigns to each $\mathbb{A}$ !-bimodule $\mathfrak{a}$, the $\mathbb{A}$ !-bimodule map

$$
\begin{aligned}
\eta_{\mathfrak{a}}: \mathfrak{a} & \rightarrow \widetilde{\mathfrak{a}}=\operatorname{Hom}_{\mathbb{A}^{0}}\left(\varphi^{00} \mathbb{A} !, \varphi^{00} \mathfrak{a}\right), \\
a & \mapsto-\cdot a,
\end{aligned}
$$

where $-\cdot a: \varphi^{00} \mathbb{A} ! \rightarrow \varphi^{00} \mathfrak{a}$ is simply $r \mapsto r a$. (This follows immediately from the identification $\varphi^{00} \mathfrak{a}=\varphi^{00} \mathbb{A}$ ! $\otimes_{\mathbb{A}} \mathfrak{a}$ and the standard description of the unit for a Hom $-\otimes$ adjunction.) We wish to examine this in the particular case $\mathfrak{a}=\mathfrak{m}$. 
Since $\varphi^{00} \mathfrak{m}=\varphi^{00} \mathbb{M} ! e=\amalg_{g} M \varphi^{0 j}$ as a left $\mathbb{A}^{0}$-module, applying (4.2) to $\varphi^{00} \mathfrak{m}$ yields $\varphi^{i i} \tilde{\mathfrak{m}} \varphi^{j j}=\operatorname{Hom}_{\mathbb{A}^{0}}\left(\mathbb{A}^{0} \varphi^{0 i}, M \varphi^{0 j}\right)$ for $j \neq 0$ and $\varphi^{i i} \tilde{\mathfrak{m}}=\amalg_{\mathcal{J}} \varphi^{i i} \widetilde{\mathfrak{m}} \varphi^{j j}$. Then

$$
\widetilde{\mathfrak{m}}=\prod_{\mathscr{J}} \varphi^{i i} \widetilde{\mathfrak{m}}=\prod_{\mathscr{J}} \coprod_{\mathscr{J}} \varphi^{i i} \widetilde{\mathfrak{m}} \varphi^{j j}
$$

and

$$
\varphi^{i i} \tilde{\mathfrak{m}} \varphi^{j j}=\operatorname{Hom}_{\mathbb{A}^{0}}\left(\mathbb{A}^{0} \varphi^{0 i}, M \varphi^{0 j}\right) \rightarrow M \varphi^{0 j}: f \mapsto f\left(\varphi^{0 i}\right)
$$

is a bijection for $j \neq 0$. Now, for $m \in \mathfrak{m}$, this means that $\eta_{\mathfrak{m}}(m)=\sum \varphi^{i i} \eta_{\mathfrak{m}}(m) \varphi^{j j}$ while $\varphi^{i i} \eta_{\mathfrak{m}}(m) \varphi^{j j}=\eta_{\mathfrak{m}}\left(\varphi^{i i} m \varphi^{j j}\right) \in \varphi^{i i} \tilde{\mathfrak{m}} \varphi^{j j}$ is the left $\mathbb{A}^{0}$-module map $\mathbb{A}^{0} \varphi^{0 i} \rightarrow$ $M \varphi^{0 j}$ determined by $\varphi^{0 i} \mapsto \varphi^{0 i} \cdot\left(\varphi^{i i} m \varphi^{j j}\right)$. We can now compute the kernel and image of $\eta_{\mathfrak{m}}$.

For the kernel, suppose that $\eta_{\mathfrak{m}}(m)=0$. Then each $\varphi^{i i} \eta_{\mathfrak{m}}(m) \varphi^{j j}=0$ and, so, $\varphi^{0 i} \cdot\left(\varphi^{i i} m \varphi^{j j}\right)=0 \in M \varphi^{0 j}$ for all $i \in \mathscr{I}$ and $j \in \mathscr{J}$. But $\varphi^{0 i} \cdot-: M \varphi^{i j} \rightarrow M \varphi^{0 j}$ is a $k$-linear isomorphism since $\mathbb{M}^{i} \rightarrow \mathbb{M}^{0}=M$ is an identity map. Hence, each $\varphi^{i i} m \varphi^{j j}=0$ and $m=\sum \varphi^{i i} m \varphi^{j j}=0$. That is, the kernel is trivial and $\eta_{\mathfrak{m}}$ is a monomorphism.

For the image, first note that $\varphi^{i i} \mathfrak{m} \varphi^{j j}=\varphi^{i i} \mathbb{M} ! e \varphi^{j j}=0$ unless $j \in i / \mathscr{J}=\{j \in$ $\mathscr{J} \mid j \geq i\}$, in which case $\varphi^{i i} \mathfrak{m} \varphi^{j j}=M \varphi^{i j}$. Thus, $\mathfrak{m}=\prod_{\mathscr{F}} \coprod_{i / \mathscr{J}} \varphi^{i i} \mathfrak{m} \varphi^{j j}$. Also, as noted above, $\widetilde{\mathfrak{m}}=\prod_{\mathscr{\digamma}} \amalg_{\mathscr{J}} \varphi^{i i} \widetilde{\mathfrak{m}} \varphi^{j j}$ and, of course, $\varphi^{i i} \eta_{\mathfrak{m}}(\mathfrak{m}) \varphi^{j j}=\eta_{\mathfrak{m}}\left(\varphi^{i i} \mathfrak{m} \varphi^{j j}\right)$. It follows that $\eta_{\mathfrak{m}}(\mathfrak{m})=\prod_{\mathscr{G}} \bigsqcup_{i / \mathcal{J}} \varphi^{i i} \eta_{\mathfrak{m}}(\mathfrak{m}) \varphi^{j j}$. Now, when $j \in i / \mathcal{J}$ the composite $k$-bimodule map

$$
\begin{aligned}
& M \varphi^{i j}=\varphi^{i i} \mathfrak{m} \varphi^{j j} \stackrel{\eta_{\mathrm{m}}}{\longrightarrow} \varphi^{i i} \widetilde{\mathfrak{m}} \varphi^{j j} \rightarrow M \varphi^{0 j} \\
& m \varphi^{i j} \mapsto-\cdot m \varphi^{i j} \mapsto \varphi^{0 i} \cdot\left(m \varphi^{i j}\right)=m \varphi^{0 j}
\end{aligned}
$$

is clearly surjective, which implies that $\eta_{\mathfrak{m}}\left(M \varphi^{i j}\right)=\varphi^{i i} \tilde{\mathfrak{m}} \varphi^{j j}$. The image of $\eta_{\mathfrak{m}}$ is thus $\eta_{\mathfrak{m}}(\mathfrak{m})=\prod_{\mathscr{F}} \bigsqcup_{i / \mathcal{g}} \varphi^{i i} \widetilde{\mathfrak{m}} \varphi^{j j}$.

For these computations we draw two conclusions. First, since the image of $\eta_{\mathfrak{m}}$ is a $k$-bimodule direct summand of $\tilde{\mathfrak{m}}$, the monomorphism $\eta_{\mathfrak{m}}$ and the exact sequence

$$
0 \rightarrow \mathfrak{m} \rightarrow \widetilde{\mathfrak{m}} \rightarrow \operatorname{cok} \eta_{\mathfrak{m}} \rightarrow 0
$$

are allowable. (In general, $\eta_{\mathfrak{a}}$ need be neither allowable or monic.) Second, $\operatorname{cok} \eta_{\mathfrak{m}}=$ $\prod_{\mathscr{J}} \varphi^{i i} \operatorname{cok} \eta_{\mathfrak{m}}$ and $\varphi^{i i} \operatorname{cok} \eta_{\mathfrak{m}} \varphi^{j j}=0$ whenever $i \leq j$. That is, in the terminology of $\S 3$, cok $\eta_{\mathfrak{m}}$ is an $\Omega_{\leq}$-hostile $\mathbb{A}$ !-bimodule. Since $\mathbb{N}$ ! is $\Omega_{\leq}$-aligned for every $\mathbb{A}$ bimodule $\mathbb{N}$, Theorem $3.9(1)$ yields

Lemma 4.4. If $\mathbb{N}$ is an $\mathbb{A}$-bimodule then $\operatorname{Ext}_{\mathbb{A} !-\mathrm{A} !}^{\bullet}\left(\mathbb{N} !, \operatorname{cok} \eta_{\mathfrak{m}}\right)=0$.

In view of (4.3) and the last lemma, we can conclude that $\operatorname{Ext}_{\mathbf{A} !-\mathrm{A} !}^{\bullet}(-!, \mathfrak{m})=0$ once we show that $\operatorname{Ext}_{\mathrm{A} !-\mathrm{A} !}^{\bullet}(-!, \tilde{\mathfrak{m}})=0$. Our proof of the latter equality involves the functor $\operatorname{Hom}_{k-\mathbf{A} !}\left(\varphi^{00} \mathbb{A} !,-\right): \mathbb{A} !-$ bimod $\rightarrow\left(\mathbb{A} !-\mathbb{A}^{0}\right)$-bimod, its left adjoint $-\otimes_{\mathbb{A}^{0}} \varphi^{00} \mathbb{A}$ !, and the counit of the adjunction. As with the previous adjoint pair, since $\varphi^{00} \mathbb{A}$ ! is projective both as a right $\mathbb{A}$ !-module and as a left $\mathbb{A}^{0}$-module, these are exact functors which preserve allowability. Note that $\operatorname{Hom}_{k \text {-A! }}\left(\varphi^{00} \mathbb{A} !,-\right)$ is naturally equivalent to $\mathfrak{a} \rightsquigarrow \mathfrak{a} \varphi^{00}$; the isomorphism $\operatorname{Hom}_{k-\mathbb{A} !}\left(\varphi^{00} \mathbb{A} !, \mathfrak{a}\right) \rightarrow \mathfrak{a} \varphi^{00}$ is simply $f \mapsto f\left(\varphi^{00}\right)$ and we shall use it to identify these two bimodules. For simplicity, we set $\widehat{\mathfrak{a}}=\mathfrak{a} \varphi^{00} \otimes_{\mathbb{A}^{0}} \varphi^{00} \mathbb{A}$ !. The counit of this adjunction is easily seen to 
be the natural transformation which assigns to each $\mathbb{A}$ !-bimodule $\mathfrak{a}$ the $\mathbb{A}$ !-bimodule map

$$
\begin{aligned}
\varepsilon_{\mathfrak{a}}: \widehat{\mathfrak{a}}=\mathfrak{a} \varphi^{00} \otimes_{\mathbb{A}^{0}} \varphi^{00} \mathbb{A} ! & \rightarrow \mathfrak{a}, \\
a \otimes r & \mapsto a r .
\end{aligned}
$$

In particular, when $\mathfrak{a}=\varphi^{00} \mathbb{N} !=\amalg \mathbb{N}^{0} \varphi^{0 i}$, we have $\mathfrak{a} \varphi^{00}=\mathbb{N}^{0} \varphi^{00}$ and $\varepsilon_{\mathfrak{a}}$ is the obvious isomorphism $\mathbb{N}^{0} \varphi^{00} \otimes_{\mathbb{A}^{0}} \amalg \mathbb{A}^{0} \varphi^{0 i} \rightarrow \amalg \mathbb{N}^{0} \varphi^{0 i}$.

LemmA 4.5. If $\mathbb{N}$ is an $\mathbb{A}$-bimodule, $\mathfrak{a}$ is an $\mathbb{A}$ !-bimodule, and $\mathfrak{a} \varphi^{00}=0$ then $\operatorname{Ext}_{\mathbb{A} !-\mathbb{A} !}^{\bullet}(\mathbb{N} !, \widetilde{\mathfrak{a}})=0$. In particular, $\operatorname{Ext}_{\mathbb{A} !-\mathbb{A} !}^{\bullet}(\mathbb{N} !, \widetilde{\mathfrak{m}})=0$.

PROOF. The first of the adjunctions establishes a bijection $\operatorname{Hom}_{\mathbb{A} !-\mathbb{A} !}(\mathbb{N} !, \mathfrak{a}) \cong$ $\operatorname{Hom}_{\mathbb{A}^{0}-\mathbb{A} !}\left(\varphi^{00} \mathbb{N} !, \varphi^{00} \mathfrak{a}\right)$ and, so, the dimension zero case will follow if we show the latter to be trivial. Suppose that $f: \varphi^{00} \mathbb{N} ! \rightarrow \varphi^{00} \mathfrak{a}$ is an $\left(\mathbb{A}^{0}\right.$ - $\left.\mathbb{A} !\right)$-bimodule map and note that $\varphi^{00} \mathbb{N} !=\left(\mathbb{N}^{0} \varphi^{00}\right) \mathbb{A} !$. Then, since $f\left(\mathbb{N}^{0} \varphi^{00}\right) \subset \varphi^{00} \mathfrak{a} \varphi^{00}=0$, we have $f=0$, as required.

For the case of dimension $n>0$, recall from Lemma 3.6 that $0 \rightarrow \varphi^{00} \mathbb{N} ! \rightarrow \mathbb{N} ! \rightarrow$ $\left(\langle\mathcal{F}\rangle^{*} \mathbb{N}\right) ! \rightarrow 0$ is an allowable short exact sequence. Hence it suffices to show that $\operatorname{Ext}_{\mathrm{A} !-\mathrm{A} !}^{n}\left(\varphi^{00} \mathbb{N} !, \tilde{\mathfrak{a}}\right)=0$ and that $\operatorname{Ext}_{\mathrm{A} !-\mathrm{A} !}^{n}\left(\left(\langle\mathscr{J}\rangle^{*} \mathbb{N}\right) !, \tilde{\mathfrak{a}}\right)=0$.

Suppose that $[\mathscr{F}] \in \operatorname{Ext}_{\mathbb{A} !-\mathrm{A} !}^{n}\left(\varphi^{00} \mathbb{N} !, \widetilde{\mathfrak{a}}\right)$. The counit of the second adjunction provides a map of extensions $\varepsilon_{\mathscr{F}}: \widehat{\mathscr{F}} \rightarrow \mathscr{F}$. Observe that $(\mathfrak{a})^{\wedge}=0$ since $\tilde{\mathfrak{a}} \varphi^{00}=$ $\operatorname{Hom}_{\mathbb{A}^{0}}\left(\varphi^{00} \mathbb{A} !, \varphi^{00} \mathfrak{a} \varphi^{00}\right)=0$. Hence the map of extensions $\varepsilon_{\mathscr{F}}: \widehat{\mathscr{F}} \rightarrow \mathscr{F}$ starts with $0 \rightarrow \tilde{\mathfrak{a}}$ and ends with the map $\varepsilon=\varepsilon_{\varphi^{00} \mathbb{N} !}:\left(\varphi^{00} \mathbb{N} !\right)^{\wedge} \rightarrow \varphi^{00} \mathbb{N}$ ! which, as was noted above, is an isomorphism. This yields $0=0 \widehat{\mathscr{F}} \equiv \mathscr{F} \varepsilon$ and, so, $[\mathscr{F} \varepsilon]=0$. Now the map

$$
\operatorname{Ext}_{\mathbb{A} !-\mathbb{A} !}^{n}\left(\varphi^{00} \mathbb{N} !, \tilde{\mathfrak{a}}\right) \rightarrow \operatorname{Ext}_{\mathbb{A} !-\mathbb{A} !}^{n}\left(\left(\varphi^{00} \mathbb{N} !\right) ; \tilde{\mathfrak{a}}\right):\left[\mathscr{F}^{\prime}\right] \mapsto\left[\mathscr{F}^{\prime} \varepsilon\right]
$$

is an isomorphism since it is induced by an isomorphism. Thus, we have $[\mathscr{F}]=0$ as well; that is, $\operatorname{Ext}_{\mathrm{A} !-\mathrm{A} !}^{n}\left(\varphi^{00} \mathbb{N} !, \widetilde{\mathfrak{a}}\right)=0$.

Next, suppose that $[\mathscr{F}] \in \operatorname{Ext}_{\mathrm{A} !-\mathbb{A} !}^{n}\left(\left(\langle\mathscr{J}\rangle^{*} \mathbb{N}\right) !, \tilde{\mathfrak{a}}\right)$. The unit of the first adjunction yields a map of extensions $\eta_{\mathscr{F}}: \mathscr{F} \rightarrow \widetilde{\mathscr{F}}$. Now, as noted earlier, $\eta=\eta_{\tilde{\mathfrak{a}}}: \widetilde{\mathfrak{a}} \rightarrow(\mathfrak{a})^{\sim}$ is an isomorphism. On the other hand, $\left(\left(\langle\mathscr{J}\rangle^{*} \mathbb{N}\right) !\right)^{\sim}=0$ since $\varphi^{00}\left(\left(\langle\mathscr{J}\rangle^{*} \mathbb{N}\right) !\right)=0$. Then, since the map $\eta_{\mathscr{F}}: \mathscr{F} \rightarrow \widetilde{\mathscr{F}}$ starts with the isomorphism $\eta$ and ends with $\left(\langle\mathscr{J}\rangle^{*} \mathbb{N}\right) ! \rightarrow 0$, we have $\eta \mathscr{F} \equiv \widetilde{\mathscr{F}} 0=0$. As before, this implies that $\mathscr{F} \equiv 0$ and, so, $\operatorname{Ext}_{\mathbb{A} !-\mathrm{A} !}^{n}\left(\left(\langle\mathscr{J}\rangle^{*} \mathbb{N}\right) !, \widetilde{\mathfrak{a}}\right)=0$. This completes the proof of the first assertion. Since $\mathfrak{m} \varphi^{00}=\mathbb{M} ! e \varphi^{00}=0$, the second then follows trivially.

COROLlary 4.6. If $\mathbb{N}$ is an $\mathbb{A}$-bimodule, then $\operatorname{Ext}_{\mathbb{A} !-\mathbb{A} !}^{\bullet}(\mathbb{N} !, \mathfrak{m})=0$.

As we remarked after (4.1), Corollaries 4.3 and 4.6 imply

COROllary 4.7. If $\mathbb{N}$ is an $\mathbb{A}$-bimodule, $M$ is an $\mathbb{A}^{0}$-bimodule, and $\mathbb{M}=$ $\langle 0\rangle_{*} M$ then $\operatorname{Ext}_{\mathbb{A}^{\prime}-\mathrm{A} !}^{\bullet !}(\mathbb{N} !, \mathbb{M} !) \cong \operatorname{Ext}_{\mathbb{A}^{0}-\mathbb{A}^{0}}^{\bullet}\left(\mathbb{N}^{0}, M\right)$.

The Second Acyclicity Theorem now follows since an $\mathbb{A}$-bimodule $\mathbb{M}$ is a 0 primitive relative injective if and only if $\mathbb{M}=\langle 0\rangle_{*} M$ for some relative injective $\mathbb{A}^{0}$-bimodule $M$, in which case $\operatorname{Ext}_{\mathbb{A}^{0}-\mathbb{A}^{0}}^{n}\left(\mathbb{N}^{0}, M\right)=0$ for $n>0$. According to Theorem 3.8, we have also established the following. 
The SPECIAL COHOMOlogy COMPARISON THEOREM. For an arbitrary diagram $\mathbb{A}$ over an arbitrary poset, $\omega^{\bullet}: \operatorname{Ext}_{\mathbb{A}-\mathbb{A}}^{\bullet}(-,-) \rightarrow \operatorname{Ext}_{\mathbb{A} !-\mathbb{A} !}^{\bullet}(-!,-!)$ is an isomorphism.

We conclude this section with a comment about the simplest nontrivial case of the foregoing, namely $\mathscr{I}=\{0<1\}$. It is easy to see that this is the only case in which $\eta_{\mathfrak{m}}$ is an isomorphism. Of course, Lemma 4.4 then becomes a triviality, the application of Theorem 3.9(1) is unnecessary, and Lemma 4.5 coincides with Corollary 4.6 .

5. Relative injectives revisited. In this section we will show that ! preserves essentially no primitive relative injectives and, more important, preserves enough relative injectives if and only if $\mathscr{I}$ is discrete. We begin by identifying the primitive relative injectives it does preserve.

LEMMA 5.1. If $i \in \mathcal{I}$ is a maximal element and $M$ is an $\mathbb{A}^{i}$-bimodule then $\left(\langle i\rangle_{*} M\right)$ ! is a relative injective $\mathbb{A} !$-bimodule if and only if $M$ is a relative injective $\mathbb{A}^{i}$-bimodule.

ProOF. To begin with, if $\left(\langle i\rangle_{*} M\right)$ ! is a relative injective $\mathbb{A}$ !-bimodule then, since ! is full and exact, $\langle i\rangle_{*} M$ is a relative injective $\mathbb{A}$-bimodule and Lemma 1.2 guarantees, in turn, that $M$ is a relative injective $\mathbb{A}^{i}$-bimodule.

For the other direction, first note that $\left(\langle i\rangle_{*} M\right) !=M \varphi^{i i}$. Now let $f: \mathfrak{a} \rightarrow M \varphi^{i i}$ and $\mathfrak{a} \mapsto \mathfrak{b}$ be $\mathbb{A}$ !-bimodule maps and assume that $\mathfrak{a} \nrightarrow \mathfrak{b}$ is allowable. Then $f\left(\left(1-\varphi^{i i}\right) \mathfrak{a}\right) \subset\left(1-\varphi^{i i}\right) M \varphi^{i i}=0$ and $f\left(\mathfrak{a}\left(1-\varphi^{i i}\right)\right) \subset M \varphi^{i i}\left(1-\varphi^{i i}\right)=0$. So $f$ is completely determined by its restriction $f^{i}: \varphi^{i i} \mathfrak{a} \varphi^{i i} \rightarrow M \varphi^{i i}$, which is an allowable $\mathbb{A}^{i}$-bimodule map. Now, due to the relative injectivity of $M$ there is an extension of $f^{i}$ to an $\mathbb{A}^{i}$-bimodule map $\hat{f}: \varphi^{i i} \mathfrak{b} \varphi^{i i} \rightarrow M \varphi^{i i}$. If we set $\hat{f}\left(\left(1-\varphi^{i i}\right) \mathfrak{b}\right)=$ $\hat{f}\left(\mathfrak{b}\left(1-\varphi^{i i}\right)\right)=0$ then, as is easily checked, $\hat{f}$ is an $\mathbb{A}$ !-bimodule map which, plainly, extends $f$.

Note that the second paragraph of the proof above could be replaced by the observation that $\{i\}$ is a filter in $\mathscr{I}$ and an appeal to Theorem 3.7. However, doing so would disguise the elementary nature of the result. In contrast to Lemma 5.1 we have

LEMMA 5.2. Let $\mathscr{I}$ be a nontrivial cone with least element 0 and let $M$ be an $\mathbb{A}^{0}$-bimodule. Then $\left(\langle 0\rangle_{*} M\right)$ ! is a relative injective $\mathbb{A}$ !-bimodule if and only if $M=0$.

ProOF. Let $\mathscr{J}$ be the filter $\{i>0\}$, set $e=\sum_{\mathcal{g}} \varphi^{j j}$, and, as in $\S 4$, set $\mathbb{M}=\langle 0\rangle_{*} M$. We consider the $\mathbb{A}$ !-bimodules $\mathfrak{a}=\varphi^{00} \mathbb{A} ! e \otimes \varphi^{00} \mathbb{A} !$ and $\mathfrak{b}=\mathbb{A} ! e \otimes \varphi^{00} \mathbb{A}$ !. The obvious inclusion $\mathfrak{a} \rightarrow \mathfrak{b}$ is allowable. We shall show that $\operatorname{Hom}_{\mathbb{A} !-\mathbf{A} !}(\mathfrak{b}, \mathbb{M} !) \rightarrow$ $\operatorname{Hom}_{\mathbf{A} !-\mathrm{A} !}(\mathfrak{a}, \mathbb{M} !)$ is not an epimorphism if $M \neq 0$; this yields the lemma.

First, for any $\mathbb{A}$ !-bimodule map $f: \mathfrak{b} \rightarrow \mathbb{M}$ ! we must have $f\left(e \otimes \varphi^{00}\right) \in e \mathbb{M} ! \varphi^{00}=$ 0 . But then $f(\mathfrak{b})=\mathbb{A} ! f\left(e \otimes \varphi^{00}\right) \mathbb{A} !=0$. That is, $\operatorname{Hom}_{\mathbb{A} !-\mathbb{A} !}(\mathfrak{b}, \mathbb{M} !)=0$.

Next, note that $\mathfrak{a}$ is naturally an $\left(\mathbb{A}^{0}-\mathbb{A}\right.$ !)-bimodule and that $\mathfrak{a}=\left(\varphi^{00} \mathbb{A} ! e \otimes\right.$ $\left.\mathbb{A}^{0} \varphi^{00}\right) \otimes_{\mathbb{A}^{0}} \varphi^{00} \mathbb{A}$ !. Also, there is an $\mathbb{A}^{0}$-bimodule isomorphism $\varphi^{00} \mathbb{A} ! e \otimes \mathbb{A}^{0} \varphi^{00} \cong$ $\mathbb{A}^{0} \otimes \amalg_{\mathcal{J}} k \otimes \mathbb{A}^{0}$, since as a left $\mathbb{A}^{0}$-module $\varphi^{00} \mathbb{A} ! e=\coprod_{\mathcal{J}} \mathbb{A}^{0} \varphi^{0 j} \cong \mathbb{A}^{0} \times \amalg_{\mathcal{g}} k$. Hence, some standard Hom $-\otimes$ adjunctions, the adjunction discussed at the outset 
of $\S 4$, and the observation that $\operatorname{Hom}_{\mathbb{A} !}\left(\varphi^{00} \mathbb{A} !, \varphi^{00} \mathbb{M} !\right)=\varphi^{00} \mathbb{M} ! \varphi^{00}=M \varphi^{00}$ yield

$$
\begin{aligned}
\operatorname{Hom}_{\mathbb{A} !-\mathbb{A} !}(\mathfrak{a}, \mathbb{M} !) & \cong \operatorname{Hom}_{\mathbf{A}^{0}-\mathbf{A} !}\left(\mathfrak{a}, \varphi^{00} \mathbb{M} !\right) \\
& \cong \operatorname{Hom}_{\mathbf{A}^{0}-\mathbf{A}^{0}}\left(\varphi^{00} \mathbb{A} ! e \otimes \mathbb{A}^{0} \varphi^{00}, \operatorname{Hom}_{\mathbf{A} !}\left(\varphi^{00} \mathbb{A} !, \varphi^{00} \mathbb{M} !\right)\right) \\
& \cong \operatorname{Hom}_{k-k}\left(\coprod_{\mathscr{J}} k, M\right) \cong \prod_{\mathcal{J}} M .
\end{aligned}
$$

Thus, if $M \neq 0$ then $\operatorname{Hom}_{\mathbf{A} !-\mathbf{A} !}(\mathfrak{a}, \mathbb{M} !) \neq 0$ and we are done.

We can now prove that! preserves remarkably few primitive relative injectives.

THEOREM 5.3. Let $\mathscr{I}$ be an arbitrary poset and let $M$ be a nonzero $\mathbb{A}^{i}$ bimodule. Then $\left(\langle i\rangle_{*} M\right)$ ! is a relative injective $\mathbb{A}$ !-bimodule if and only if $i$ is maximal in $\mathscr{I}$ and $M$ is a relative injective $\mathbb{A}^{i}$-bimodule.

Proof. The "if" statement was established as Lemma 5.1. For the "only if" statement we set $\mathcal{J}=\{j \geq i\}$, abbreviate $\langle i\rangle_{*} M$ to $\mathbb{M}$, and assume that $\mathbb{M}$ ! is a relative injective $\mathbb{A}$ !-bimodule. Observe that, in the notation of $\S 3, \mathbb{M}=\langle\mathscr{J}\rangle_{*} \mathbb{M}^{\prime}$ where $\mathbb{M}^{\prime}=\langle\mathscr{J}\rangle^{*} \mathbb{M}$. Also, $\mathbb{M}^{\prime}$ !, when viewed as an $\mathbb{A}$ !-bimodule through $\mathbb{A}$ ! $\rightarrow$ $\left(\langle\mathcal{f}\rangle^{*} \mathbb{A}\right)$ !, is isomorphic to $\mathbb{M}$ !. Now suppose that $f: \mathfrak{a} \rightarrow \mathbb{M}^{\prime}$ ! and $\mathfrak{a} \rightarrow \mathfrak{b}$ are $\left(\langle\mathcal{F}\rangle^{*} \mathbb{A}\right)$ !-bimodule maps, with the latter map being allowable. Then, considered as an $\mathbb{A}$ !-bimodule map, $f$ has an extension to an $\mathbb{A}$ !-bimodule map $\mathfrak{b} \rightarrow \mathbb{M}$ ! $=\mathbb{M}^{\prime}$ !. Since this extension is certainly a $\left(\langle\mathscr{J}\rangle^{*} \mathbb{A}\right)$ !-bimodule map, we have shown $\mathbb{M}^{\prime}$ ! to be a relative injective $\left(\langle\mathscr{J}\rangle^{*} \mathbb{A}\right)$ !-bimodule. But $\mathbb{M}^{\prime}$ is the right inflation of $M$ from $\mathbb{A}^{i}$-bimod to $\left(\langle\mathscr{J}\rangle^{*} \mathbb{A}\right)$ !-bimod and $\mathscr{J}$ is a cone with least element $i$. Hence Lemma 5.2 requires that $\mathcal{J}=\{i\}$ and, so, $i$ is maximal in $\mathscr{I}$. Now Lemma 5.1 implies that $M$ is a relative injective $\mathbb{A}^{i}$-bimodule.

Suppose that $\mathscr{I}$ is not discrete, that $\mathbb{E}$ is an $i$-primitive relative injective for some nonmaximal $i \in \mathscr{I}$, and that $\mathbb{E} \nrightarrow \mathbb{E}_{0}$ is an allowable $\mathbb{A}$-bimodule monomorphism. Then $\mathbb{E} \nrightarrow \mathbb{E}_{0}$ splits and, so, $\mathbb{E}_{0}=\mathbb{E} \oplus \mathbb{E}^{\prime}$ for some $\mathbb{A}$-bimodule $\mathbb{E}^{\prime}$. But then, since $\mathbb{E}$ ! is not a relative injective $\mathbb{A}$ !-bimodule, $\mathbb{E}_{0}$ ! cannot be a relative injective either. In particular, $\mathbb{E}$ itself can have no relative injective allowable resolution $0 \rightarrow \mathbb{E} \rightarrow \mathbb{E}_{\bullet}$ in $\mathbb{A}$-bimod with the property that $0 \rightarrow \mathbb{E} ! \rightarrow \mathbb{E}_{\bullet}$ ! is such a resolution in $\mathbb{A}$ !-bimod. Combining this observation with Lemma 5.1 yields

THEOREM 5.4. ! preserves enough relative injectives if and only if $\mathscr{F}$ is discrete.

The considerations of this section dualize in a reasonably straightforward fashion and, consequently, ! does not preserve enough relative projectives.

6. Hochschild cohomology. For an associative $k$-algebra $A$ the Hochschild cohomology functors on $A$-bimod may be defined by $H^{\bullet}(A,-)=\operatorname{Ext}_{A-A}^{\bullet}(A,-)$. So, too, we may define the Hochschild cohomology functors on $\mathbb{A}$-bimod by $H^{\bullet}(\mathbb{A},-)=$ $\operatorname{Ext}_{\mathbb{A}-\mathbb{A}}^{\bullet}(\mathbb{A},-)$. The CCT then implies that $H^{\bullet}(\mathbb{A},-) \cong H^{\bullet}(\mathbb{A} !,-!)$. When $\mathscr{I}$ is finite the latter isomorphism is easier to obtain than the full CCT. In fact, we shall give a cochain map which induces the isomorphism. This involves, amongst other things, providing a cochain complex $C^{\bullet}(\mathbb{A},-)$ for which $H\left(C^{\bullet}(\mathbb{A},-)\right)=H^{\bullet}(\mathbb{A},-)$. We begin with a closer look at $\operatorname{Ext}_{A-A}^{\bullet}(-,-)$, in general, and $\operatorname{Ext}_{\mathrm{A} !-\mathrm{A} !}^{\bullet}(-,-)$, in particular. 
If $A$ is a $k$-algebra and $R \subset A$ is a subalgebra then a map $f: N \rightarrow M$ in $A$-bimod is called $R$-allowable if there is an $R$-bimodule map $\lambda: M \rightarrow N$ for which $f \lambda f=f$. An extension $\mathscr{E}$ in $A$-bimod is $R$-allowable if every map appearing in $\mathscr{E}$ is $R$-allowable. Such extensions form the foundation for an $R$ relative Yoneda cohomology $\operatorname{Ext}_{A-A, R}^{\circ}(-,-)$ in the usual way. Note that the definition of $k$-allowable is precisely the definition of allowable given in $\S 1$ and, so, $\operatorname{Ext}_{A-A, k}^{\bullet}(-,-)=\operatorname{Ext}_{A-A}^{\circ}(-,-)$. Similarly, we define $R$-relative projective, etc., in an obvious way, generalizing the definitions of $\S 1$ for the case $R=k$. Since any $R$-allowable map is, preforce, allowable there is a natural transformation $\operatorname{Ext}_{A-A, R}^{\bullet}(-,-) \rightarrow \operatorname{Ext}_{A-A}^{\bullet}(-,-):[\mathscr{E}] \mapsto[\mathscr{E}]$. Now, Theorem 1.2 of $[$ GS4] asserts

If $R$ is separable (over $k$ ) then

$\operatorname{Ext}_{A-A, R}^{\bullet}(-,-) \rightarrow \operatorname{Ext}_{A-A}^{\bullet}(-,-)$

is an isomorphism.

We set $H^{\bullet}(A, R ;-)=\operatorname{Ext}_{A-A, R}^{\bullet}(A,-)$. To find cochains for $H^{\bullet}(A, R ;-)$ we use a suitable $R$-relative projective allowable resolution, $\mathscr{P}_{\bullet} \rightarrow A \rightarrow 0$, of $A$ in $A$-bimod, namely: $\mathscr{P}_{n}=A \otimes_{R} A^{\otimes_{R^{n}}} \otimes_{R} A$, the tensor product (over $R$ ) of $n+2$ factors of $A$. The boundary $\partial^{h}$ is given by the formula $\partial^{h}\left(a_{0} \otimes \cdots \otimes a_{n+1}\right)=\sum(-1)^{i} \cdots \otimes$ $a_{i} a_{i+1} \otimes \cdots$. (The superscript " $h$ " designates Hochschild.) To obtain a normalized resolution, $\overline{\mathscr{P}}_{\bullet} \rightarrow A \rightarrow 0$, replace all but the first and last factor of $A$ in $\mathscr{P}_{n}$ by $A / R$. Now the $R$-relative cochain complex is $C^{\bullet}(A, R ;-)=\operatorname{Hom}_{A-A}\left(\mathscr{P}_{\bullet},-\right)$. It is then immediate that $C^{n}(A, R ;-)=\operatorname{Hom}_{A-A}\left(\mathscr{P}_{n},-\right)=\operatorname{Hom}_{R-R}\left(A^{\otimes_{R^{n}}},-\right)$ consists of functions of $n$ variables which are $k$-multilinear and satisfy

$$
\begin{aligned}
f\left(r a_{1}, \ldots, a_{n}\right) & =r f\left(a_{1}, \ldots, a_{n}\right), \\
f\left(a_{1}, \ldots, a_{n} r\right) & =f\left(a_{1}, \ldots, a_{n}\right) r \\
f\left(\ldots, a_{i} r, a_{i+1}, \ldots\right) & =f\left(\ldots, a_{i}, r a_{i+1}, \ldots\right)
\end{aligned}
$$

for $a_{1}, \ldots, a_{n} \in A$ and $r \in R$. The normalized $R$-relative cochain complex $\bar{C}^{\bullet}(A, R ;-)=\operatorname{Hom}_{A-A}\left(\overline{\mathscr{P}}_{\bullet},-\right)$ is the subcomplex of functions satisfying the further condition

$$
f\left(a_{1}, \cdots, a_{n}\right)=0 \quad \text { if any } a_{i} \in R .
$$

The Hochschild coboundary $\delta_{h}$ is given by $\delta_{h} f=f \partial^{h}$. We then have $H^{\bullet}(A, R ;-)=$ $H\left(C^{\bullet}(A, R ;-)\right)=H(\bar{C} \bullet(A, R ;-))$. Note that when $R=k$ the conditions in (6.2) follow from the $k$-multilinearity of $f$. We shall abbreviate $C^{\bullet}(A, k ;-)$ to $C^{\bullet}(A,-)$, etc. In a moment we shall examine this in the case $A=\mathbb{A}$ !.

In $[$ GS1, $\S 17]$ we define the strict cochain complex $C_{\boldsymbol{s}}^{\bullet}(\mathbb{A} !,-)$ as a subcomplex of $C^{\bullet}(\mathbb{A} !,-)$ in the following way: if $\mathfrak{b}$ is an $\mathbb{A}$ !-bimodule and $n>0$ then $f \in C^{n}(\mathbb{A} !, \mathfrak{b})$ is strict if

$$
\begin{aligned}
& f\left(a_{1} \varphi^{i_{1} j_{1}}, \ldots, a_{n} \varphi^{i_{n} j_{n}}\right)=0 \text { if } j_{p} \neq i_{p+1} \text { for some } p \leq n-1, \\
& f\left(a_{1} \varphi^{i_{1} i_{2}}, a_{2} \varphi^{i_{2} i_{3}}, \ldots, a_{n} \varphi^{i_{n} i_{n+1}}\right) \in \varphi^{i_{1} i_{1}} \mathfrak{b} \varphi^{i_{n+1} i_{n+1}}, \text { and } \\
& f\left(x_{1}, \ldots, x_{n}\right)=0 \text { if any } x_{i}=\varphi^{j j} \text { for some } j .
\end{aligned}
$$

We set $C_{s}^{0}(\mathbb{A} !, \mathfrak{b})=\prod_{\mathscr{I}} \varphi^{i i} \mathfrak{b} \varphi^{i i}$ and write $H_{s}^{\bullet}(\mathbb{A} !,-)$ for $H\left(C_{s}^{\bullet}(\mathbb{A} !,-)\right)$.

Observe that $\mathbb{A}$ ! contains $S=\prod_{\mathscr{F}} k \varphi^{i i}$ as a subalgebra. 
LEMMA 6.1. If $\mathscr{I}$ is finite then $C_{s}^{\bullet}(\mathbb{A} !,-)=\bar{C} \bullet(\mathbb{A} !, S ;-)$.

PROOF. Suppose that $f \in C_{s}^{n}(\mathbb{A} !, \mathfrak{b})$ for some $n>0$. Owing to the finiteness of $\mathscr{I}$ we see that (6.2) holds for $f$ if and only if it holds whenever $a_{p} \in \mathbb{A}^{i_{p}} \varphi^{i_{p} j_{p}}$ for $1 \leq p \leq n$ and $r=\varphi^{i i}$. But the latter follows immediately from the first two conditions of (6.4). Likewise, (6.3) follows from the finiteness of $\mathscr{I}$ and the third condition of (6.4). Thus, we have $C_{s}^{n}(\mathbb{A} !,-) \subset \bar{C}^{n}(\mathbb{A} !, S ;-)$ when $n>0$. For the reverse inclusion, we obtain the first condition of (6.4) from the third condition of (6.2) and the observation that $a_{r} \varphi^{i_{r} j_{r}}=a_{r} \varphi^{i_{r} j_{r}} \varphi^{j_{r} j_{r}}$ while $\varphi^{j_{r} j_{r}} a_{r+1} \varphi^{i_{r+1} j_{r+1}}=0$ if $j_{r} \neq i_{r+1}$. The second condition of (6.4) follows from the first two of (6.2) by using the identities $a_{1} \varphi^{i_{1} i_{2}}=\varphi^{i_{1} i_{1}} a_{1} \varphi^{i_{2} i_{2}}$ and $a_{n} \varphi^{i_{n} i_{n+1}}=a_{n} \varphi^{i_{n} i_{n+1}} \varphi^{i_{n+1} i_{n+1}}$. The third condition of (6.4) is a special case of (6.3).

Finally, for the case of dimension zero, note first that

$$
\bar{C}^{0}(\mathbb{A} !, S ; \mathfrak{b})=\operatorname{Hom}_{A-A}\left(A \otimes_{S} A, \mathfrak{b}\right)=\operatorname{Hom}_{S}(S, \mathfrak{b}) .
$$

Now, since $\mathscr{I}$ is finite, the $k$-bimodule decomposition of $S$ is $S=\prod_{\mathscr{I}} k \varphi^{i i}=$ $\amalg_{\mathscr{I}} k \varphi^{i i}$. This is immediately seen to be an $S$-bimodule decomposition as well and, so, $\operatorname{Hom}_{S}(S, \mathfrak{b})=\prod_{\mathscr{I}} \operatorname{Hom}_{S}\left(k \varphi^{i i}, \mathfrak{b}\right)$. Also, for $f \in \operatorname{Hom}_{S}\left(k \varphi^{i i}, \mathfrak{b}\right)$ we have $f\left(\varphi^{i i}\right) \in \varphi^{i i} \mathfrak{b} \varphi^{i i}$ since $\varphi^{i i}$ is idempotent. It follows that $\operatorname{Hom}_{S}(S, \mathfrak{b})=\prod_{\mathscr{F}} \varphi^{i i} \mathfrak{b} \varphi^{i i}$ $=C_{S}^{0}(\mathbb{A} !, \mathfrak{b})$.

Having focused attention on the subalgebra $S$ it is natural, in view of (6.1), to ask whether $S$ is separable.

LEMMA 6.2. $S$ is separable if and only if $\mathcal{I}$ is finite.

ProOF. If $\mathscr{I}$ is finite then $\sum \varphi^{i i} \otimes \varphi^{i i} \in S \otimes S^{\mathrm{op}}$ is a separability idempotent. Now suppose that $S$ is separable and let $\mathscr{M} \subset k$ be a maximal ideal. Then $\prod_{\mathscr{f}}(k / \mathscr{M}) \varphi^{i i}=(k / \mathscr{M}) \otimes S$ is a separable $(k / \mathscr{M})$-algebra. It is also a projective left $(k / \mathscr{M})$-module since $k / \mathscr{M}$ is a field. A theorem of Villamayor and Zelinsky [VZ] then implies it is finitely generated, from which it follows that $\mathscr{I}$ is finite.

The following theorem is a trivial application of the foregoing. Its value lies in the fact that it asserts that the Hochschild cohomology of $\mathbb{A}$ ! frequently can be computed using a "thinner" cochain complex than the usual one.

THEOREM 6.3. When $\mathscr{I}$ is finite $H^{\bullet}(\mathbb{A} !,-)=H^{\bullet}(\mathbb{A} !, S ;-)=H_{s}^{\bullet}(\mathbb{A} !,-)$.

We proved this-but only for the case in which the coefficient bimodule has the form $\mathbb{M}$ !- -in [GS1, §17]. Our proof there invoked the CCT because we had not yet recognized the strict cochains for what they are, namely $S$-relative cochains. The finiteness hypothesis in Theorem 6.3 can be removed if we restrict attention to aligned $\mathbb{A}$ !-bimodules, (defined in $\S 3$ ). We shall derive this as a consequence of a version of (6.1) for aligned algebras. First we need

LeMma 6.4. Let $\mathscr{I}$ and $A$ be, respectively, a set and an $\mathscr{I}$-aligned algebra. Suppose that $f: N \rightarrow M$ is an $\Omega$-aligned map between $\Omega$-aligned $A$-bimodules for some $\Omega \subset \mathscr{I} \times \mathscr{I}$. Then $f$ is allowable if and only if it is $R$-allowable, where $R=\prod_{\mathcal{g}} k e^{i}$.

Proof. One implication is trivial. For the other, suppose that $\lambda: M \rightarrow N$ is a $k$-bimodule map satisfying $f \lambda f=f$. We need to produce an $R$-bimodule map $\hat{\lambda}: M \rightarrow N$ with the same property. Fix $i \in \mathscr{I}$ and $m \in M$. Then, since $e^{i} m e^{j}=0$ 
for almost all $j \in \mathscr{J}$, it follows that $\lambda\left(e^{i} m e^{j}\right)=0$ for almost all $j \in \mathscr{J}$, and, so, $e^{i} \lambda\left(e^{i} m e^{j}\right) e^{j}=0$ for almost all $j \in \mathscr{J}$. Hence, using the $\Omega$-alignment of $N$, we can define a $k$-bimodule map $\hat{\lambda}: M \rightarrow N$ by $\hat{\lambda}(m)=\sum_{i} \sum_{j} e^{i} \lambda\left(e^{i} m e^{j}\right) e^{j}$. We shall show that this is the desired $R$-bimodule map. First, from the alignment of $f$, the relation $f \lambda f=f$, and the idempotence of $e^{i}$, we find that

$$
\begin{aligned}
f \hat{\lambda} f(m) & =f\left(\sum_{i} \sum_{j} e^{i} \lambda\left(e^{i} f(m) e^{j}\right)\right) e^{j}=\sum_{i} \sum_{j} f\left(e^{i} \lambda\left(e^{i} f(m) e^{j}\right) e^{j}\right) \\
& =\sum_{i} \sum_{j} e^{i} f \lambda f\left(e^{i} m e^{j}\right) e^{j}=\sum_{i} \sum_{j} e^{i} f\left(e^{i} m e^{j}\right) e^{j}=\sum_{i} \sum_{j} e^{i} f(m) e^{j} .
\end{aligned}
$$

But, due to the $\Omega$-alignment of $N$, the latter expression is just $f(m)$; that is, $f \hat{\lambda} f=f$, as required.

Now suppose that $r=\sum_{j} r_{j} e^{j} \in R$. Then the $k$-linearity of $\lambda$, the relation $r e^{j}=e^{j} r=r_{j} e^{j}=e^{j} r_{j}$, and (infinite) distributivity imply that

$$
\begin{aligned}
\hat{\lambda}(m r) & =\sum_{i} \sum_{j} e^{i} \lambda\left(e^{i} m r e^{j}\right) e^{j}=\sum_{i} \sum_{j} e^{i} \lambda\left(e^{i} m r_{j} e^{j}\right) e^{j} \\
& =\sum_{i} \sum_{j} e^{i} \lambda\left(e^{i} m e^{j}\right) r_{j} e^{j}=\sum_{i} \sum_{j} e^{i} \lambda\left(e^{i} m e^{j}\right) e^{j} r \\
& =\left(\sum_{i} \sum_{j} e^{i} \lambda\left(e^{i} m e^{j}\right) e^{j}\right) r=\hat{\lambda}(m) r .
\end{aligned}
$$

Thus, $\hat{\lambda}$ is a right $R$-module map. The proof that it is left $R$-linear is similar.

THEOREM 6.5. Suppose $A$ is an $\mathscr{I}$-aligned algebra and that $N$ and $M$ are $\Omega$-aligned A-bimodules for some $\Omega \subset \mathscr{I} \times \mathscr{I}$. Let $R=\prod_{\mathscr{I}} k e^{i}$. Then

$$
\operatorname{Ext}_{A-A, R}^{\bullet}(N, M) \rightarrow \operatorname{Ext}_{A-A}^{\bullet}(N, M):[\mathscr{E}] \mapsto[\mathscr{E}]
$$

is an isomorphism.

Proof. According to the last lemma and Theorem 3.9(2) the map is an epimorphism. Now the proof of Theorem 3.9 applies equally well to $\operatorname{Ext}_{A-A, R}^{\bullet}(-,-)$. Also, if $\mathscr{E}_{1}$ and $\mathscr{E}_{2}$ are $\Omega$-aligned $R$-allowable extensions and $\mathscr{E}_{1} \leftarrow \mathscr{F} \rightarrow \mathscr{E}_{2}$ is a congruence of allowable extensions then $\mathscr{F}_{\Omega}$ is $\Omega$-aligned and the last lemma insures that $\mathscr{E}_{1} \leftarrow \mathscr{F}_{\Omega} \rightarrow \mathscr{E}_{2}$ is a congruence of $R$-allowable extensions. Hence the map is a monomorphism.

Note that when $\mathscr{J}$ is finite Theorem 6.5 is a special case of (6.1) since then $R$ is separable, $A$ is $\mathcal{I}$-aligned, and every $A$-bimodule is $\Omega_{\infty}$-aligned. The last two results generalize to the case in which $R$ is a product of separable subalgebras in $A$. We do not know to what extent they hold when $R$ is replaced by other inverse limits of separable subalgebras.

If $\mathbb{A}$ is a diagram over a poset $\mathscr{I}$ and $\mathbb{M}$ is an $\mathbb{A}$-bimodule then $\mathbb{A}$ ! is $\mathscr{I}$-aligned

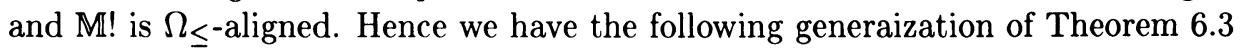

COROllaRY 6.6. $H^{\bullet}(\mathbb{A} !,-!)=H^{\bullet}(\mathbb{A} !, S ;-$ !) for any diagram $\mathbb{A}$ over any poset. 
In [GS1, §7] we introduce a Hochschild cochain complex $C^{\bullet}(\mathbb{A},-)$ for diagrams. Describing it requires some notation. Let $\Sigma$ be the nerve of $\mathscr{J}$. This is the simplicial complex whose set of $p$-simplices, $\Sigma_{p}$, consists of (proper) chains in $\mathscr{J}$ of length $p+1$. The boundary of a $p$-simplex $\sigma=\left(i_{0}<\cdots<i_{p}\right)$ is given by $\partial \sigma=\sum(-1)^{r} \sigma_{r}$ where $\sigma_{r}$ is the $(p-1)$-simplex which results from the omission of $i_{r}$. We occasionally write $(-1)^{\sigma}$ to mean $(-1)^{p}$ when $\sigma$ is a $p$-simplex. Also, for $\sigma=\left(i_{0}<\cdots<i_{p}\right)$, we set $d \sigma=i_{0}$ and $c \sigma=i_{p}$.

Now let $\mathbb{M}$ be an $\mathbb{A}$-bimodule. We define the 0 -cochains by $C^{0}(\mathbb{A}, \mathbb{M})=\prod \mathbb{M}^{i}$. For $n>0$ the $n$-cochain group is

$$
C^{n}(\mathbb{A}, \mathbb{M})=\prod_{p+q=n} \prod_{\operatorname{dim} \sigma=p} C^{q}\left(\mathbb{A}^{c \sigma}, \mathbb{M}^{d \sigma}\right)
$$

Thus an $n$-cochain is a collection of Hochschild cochains indexed by the simplices of $\Sigma$; we denote an $n$-cochain by $\Gamma=\left\{\Gamma^{\sigma}\right\}$. When $\Gamma$ is an $n$-cochain and $\sigma=\left(i_{0}<\right.$ $\left.\cdots<i_{p}\right)$ is a $p$-simplex, we set $q=n-p+1$ and define $\Gamma^{\partial \sigma} \in C^{q}\left(\mathbb{A}^{i_{p}}, \mathbb{M}^{i_{0}}\right)$ by

$$
\Gamma^{\partial \sigma}=T^{i_{0} i_{1}} \Gamma^{\sigma_{0}}-\Gamma^{\sigma_{1}}+\Gamma^{\sigma_{2}}-\cdots+(-1)^{p} \Gamma^{\sigma_{p}} \varphi^{i_{p-1} i_{p}} .
$$

The last summand in the expression above is defined by $\Gamma^{\sigma_{p}} \varphi^{i_{p-1} i_{p}}\left(a_{1}, \ldots, a_{q}\right)=$ $\Gamma^{\sigma_{p}}\left(\varphi a_{1}, \ldots, \varphi a_{q}\right)$ for $a_{1}, \ldots, a_{q} \in \mathbb{A}^{i_{p}} ;$ the first is simply the composite of the map $T^{i_{0} i_{1}}: \mathbb{M}^{i_{1}} \rightarrow \mathbb{M}^{i_{0}}$ with the cochain $\Gamma^{\sigma_{0}}$. The coboundary $\delta$ in $C^{\bullet}(\mathbb{A}, \mathbb{M})$ is then given by

$$
(\delta \Gamma)^{\sigma}=\Gamma^{\partial \sigma}+(-1)^{\sigma} \delta_{h} \Gamma^{\sigma}
$$

[Recall that $\delta_{h}$ is the Hochschild coboundary introduced after (6.3); if $\Gamma$ is an $n$ cochain, $\sigma$ is an $(n+1)$-simplex, and $\tilde{\sigma}$ is a 0 -simplex then we interpret $\Gamma^{\sigma}$ and $\Gamma^{\tilde{\sigma}}$ as 0.] The normalized cochain complex $\bar{C} \bullet(\mathbb{A}, \mathbb{M})$ is the subcomplex obtained by replacing the Hochschild cochain groups in (6.5) with the corresponding normalized cochain groups.

In $[\mathbf{G S 1}, \S 7]$ we prove that $H\left(\bar{C}^{\bullet}(\mathbb{A},-)\right)=H\left(C^{\bullet}(\mathbb{A},-)\right)=H^{\bullet}(\mathbb{A},-)$, the latter of which we defined to be $\operatorname{Ext}_{\mathbb{A}-\mathbb{A}}^{\bullet}(\mathbb{A},-)$ at the outset of this section. We sould remark that at first glance $C^{\bullet}(\mathbb{A},-)$ does not appear to arise from a relative projective allowable resolution of $\mathbb{A}$. Nonetheless, it does. The resolution is given in [GS5] and is a special case of construction used there to show that "the cohomology (of diagrams) is invariant under barycentric subdivision (of small categories)." [See the concluding remarks of $\S 2$.]

Having cochains in hand for $H^{\bullet}(\mathbb{A},-)$ and $H^{\bullet}(\mathbb{A} !,-!)$ it is natural to seek cochain maps which effect the isomorphism guaranteed by the CCT. We begin with

$$
\tau^{\bullet}: \bar{C}^{\bullet}(\mathbb{A},-) \rightarrow \bar{C}^{\bullet}(\mathbb{A} !, S ;-!)
$$

A bit of notation will make the formula concise. First, if $A$ is any $k$-algebra then there is a multiplication cochain $\pi \in C^{n}(A, A)$ for every $n>0$, namely $\pi\left(a_{1}, \ldots, a_{n}\right)=a_{1} \cdots a_{n}$. Second, if $f \in C^{\bullet}\left(\mathbb{A}^{p}, \mathbb{M}^{q}\right)$ and $p \leq i_{1}, \ldots, i_{n}$ then we interpret $f\left(a_{1}, \ldots, a_{n}\right)$ for $a_{r} \in \mathbb{A}^{i_{r}}$ in the only reasonable fashion, namely $f\left(a_{1}, \ldots, a_{n}\right)=f\left(\varphi^{p i_{1}}\left(a_{1}\right), \ldots, \varphi^{p i_{n}}\left(a_{n}\right)\right)$. Last if $\sigma=\left(i_{0} \leq \cdots \leq i_{n}\right)$ is degenerate, i.e. if $i_{r}=i_{r+1}$ for some $r$, we interpret $\Gamma^{\sigma}$ as 0 . With these conventions we 
define $\tau \Gamma \in \bar{C}^{n}(\mathbb{A} !, S ; \mathbb{M} !)$ for $\Gamma \in \bar{C}^{n}(\mathbb{A}, \mathbb{M})$ and $n>0$ by

$$
\begin{aligned}
\tau \Gamma\left(a_{1} \varphi^{i_{1} i_{2}},\right. & \left.a_{2} \varphi^{i_{2} i_{3}}, \ldots, a_{n} \varphi^{i_{n} i_{n+1}}\right) \\
& =\sum_{r=1}^{n+1} \pi\left(a_{1}, \ldots, a_{r}\right) \Gamma^{\left(i_{1} \leq \cdots \leq i_{r}\right)}\left(a_{r+1}, \ldots, a_{n}\right) \varphi^{i_{1} i_{n+1}},
\end{aligned}
$$

where $a_{r} \in \mathbb{A}^{i_{r}}$. This extends to $\mathbb{A}$ ! by (infinite) linearity. We set $\tau_{0}=$ the identity map.

Next we define $\hat{\tau}^{\bullet}: \bar{C}^{\bullet}(\mathbb{A} !, S ;-!) \rightarrow \bar{C}^{\bullet}(\mathbb{A},-)$. The definition uses shuffle products. A shuffle of $\left(a_{1}, \ldots, a_{r}\right)$ through $\left(a_{r+1}, \ldots, a_{n}\right)$ is simply a permutation of $a_{1}, \ldots, a_{n}$ which preserves the orders in $\left(a_{1}, \ldots, a_{r}\right)$ and $\left(a_{r+1}, \ldots, a_{n}\right)$. The shuffle product is then the formal sum $\left(a_{1}, \ldots, a_{r}\right) *\left(a_{r+1}, \ldots, a_{n}\right)=\sum(-1)^{\nu}\left(a_{\nu 1}, \ldots, a_{\nu n}\right)$ where $\nu$ ranges over the shuffles. We modify this slightly for diagram rings. First, to streamline the formulae we shall use the isomorphism $\mathbb{A}^{i} \rightarrow \mathbb{A}^{i} \varphi^{i i}: a \mapsto a \varphi^{i i}$ to identify $\mathbb{A}^{i} \varphi^{i i} \subset \mathbb{A}$ ! with $\mathbb{A}^{i}$. Now, if $a_{1}, \ldots, a_{n-p} \in \mathbb{A}^{i_{p}}$ then in $\left(\varphi^{i_{1} i_{2}}, \ldots, \varphi^{i_{p-1} i_{p}}\right) *$ $\left(a_{1}, \ldots, a_{n-p}\right)$ we require each $\varphi^{i j}$ to "operate" on each $a_{r}$ it passes. So, for example, $\left(\varphi^{i_{1} i_{2}}\right) *\left(a_{1}\right)=\left(\varphi^{i_{1} i_{2}}, a_{1}\right)-\left(\varphi^{i_{1} i_{2}}\left(a_{1}\right), \varphi^{i_{1} i_{2}}\right)$. Evaluation of an $n$-cochain $f$ on a shuffle product is interpreted in the obvious way, e.g., $f\left(\left(\varphi^{i_{1} i_{2}}\right)_{*}\left(a_{1}\right)\right)=$ $f\left(\varphi^{i_{1} i_{2}}, a_{1}\right)-f\left(\varphi^{i_{1} i_{2}}\left(a_{1}\right), \varphi^{i_{1} i_{2}}\right)$. Now, for $f \in \bar{C}^{n}(\mathbb{A} !, S ; \mathbb{M} !)$ and $n>0$ we define $\hat{\tau} f \in \bar{C}^{n}(\mathbb{A}, \mathbb{M})$ as follows: if $\sigma=\left(i_{0}<\cdots<i_{p}\right)$ is a $p$-simplex for some $p \leq n$ and $a_{1}, \ldots, a_{n-p} \in \mathbb{A}^{i_{p}}$ then

$$
\left((\hat{\tau} f)^{\sigma}\left(a_{1}, \ldots, a_{n-p}\right)\right) \varphi^{i_{o} i_{p}}=f\left(\left(\varphi^{i_{0} i_{1}}, \ldots, \varphi^{i_{p-1} i_{p}}\right) *\left(a_{1}, \ldots, a_{n-p}\right)\right) .
$$

Both $\tau^{\bullet}$ and $\hat{\tau}^{\bullet}$ are readily seen to be natural in the coefficient bimodule $\mathbb{M}$. Verifying that they are indeed cochain maps requires tedious but uneventful computation, which we omit. It is essentially immediate that $\hat{\tau}^{\bullet} \tau^{\bullet}$ is the identity. Also, a simple computation reveals that

$$
\begin{aligned}
& \tau \hat{\tau} f\left(a_{1} \varphi^{i_{1} i_{2}}, a_{2} \varphi^{i_{2} i_{3}}, \ldots, a_{n} \varphi^{i_{n} i_{n+1}}\right) \\
& \quad=\sum \pi\left(a_{1}, \ldots, a_{r}\right) f\left(\left(\varphi^{i_{1} i_{2}}, \ldots, \varphi^{i_{r}, i_{r+1}}\right) *\left(a_{r+1}, \ldots, a_{n}\right)\right) \varphi^{i_{r+1} i_{n+1}} .
\end{aligned}
$$

We have defined $\tau^{\bullet} \hat{\tau}^{\bullet}$ to be a cochain map from $\bar{C}^{\bullet}(\mathbb{A}$ !, $S ;-$ !) to itself. However, (6.6) shows that when $\mathscr{I}$ is finite $\tau^{\bullet} \hat{\tau}^{\bullet}$ is defined if we use an arbitrary $\mathbb{A}$ !-bimodule $\mathfrak{b}$ as the coefficient bimodule. Thus, when $\mathscr{I}$ is finite $\tau^{\bullet} \hat{\tau}^{\bullet}$ is a natural transformation from $\bar{C}^{\bullet}(\mathbb{A} !, S ;-)=\operatorname{Hom}_{\mathbb{A} !-\mathrm{A} !}\left(\overline{\mathscr{P}}_{\bullet},-\right)$ to itself. Yoneda's lemma then implies that $\tau^{\bullet} \hat{\tau}^{\bullet}$ is induced by a unique chain map $t_{\bullet}: \overline{\mathscr{P}}_{\bullet} \rightarrow \overline{\mathscr{P}}_{\bullet}$. (In fact, an examination of (6.6) quickly leads to a formula for $t_{\bullet}$. We shall not need it.) Since $\tau^{0} \hat{\tau}^{0}$ is the identity map we must have $t_{0}=$ Id. But then $t_{\bullet}$ and $I_{\bullet}: \overline{\mathscr{P}}_{\bullet} \rightarrow \overline{\mathscr{P}}_{\bullet}$ are both liftings of the identity map $\mathbb{A} ! \rightarrow \mathbb{A}$ ! to a map of $S$-relative projective $S$-allowable resolutions of $\mathbb{A}$ !. It follows that they are chain homotopic: $t_{\bullet} \sim \mathrm{Id}_{\bullet}$. Hence, $\tau^{\bullet} \hat{\tau}^{\bullet} \sim \operatorname{Id}^{\bullet}$ and $H\left(\tau^{\bullet} \hat{\tau}^{\bullet}\right)=\operatorname{Id}$. Since $\hat{\tau}^{\bullet} \tau^{\bullet}$ is the identity we have proved

THEOREM 6.7. If $\mathscr{I}$ is finite then $H\left(\tau^{\bullet}\right)$ is an isomorphism $H^{\bullet}(\mathbb{A},-) \rightarrow$ $H^{\bullet}(\mathbb{A} !, S ;-!)$.

Let $\tilde{\tau}^{\bullet}$ be the composition of $\tau^{\bullet}$ with the inclusion of complexes $\bar{C} \bullet(\mathbb{A} !, S ;-) \hookrightarrow$ $C^{\bullet}(\mathbb{A} !,-)$. Then, as is easily checked, $H\left(\tilde{\tau}^{0}\right): \operatorname{Hom}_{\mathbf{A}-\mathbf{A}}(\mathbb{A},-) \rightarrow \operatorname{Hom}_{\mathbf{A} !-\mathbb{A} !}(\mathbb{A} !,-!)$ is just $\eta \mapsto \eta$ !, which was defined to be $\omega^{0}$ in $\S 2$. Hence $\omega^{\bullet}$ and $H\left(\tilde{\tau}^{\bullet}\right)$ both extend $\omega^{0}$. The universality of $H^{\bullet}(\mathbb{A},-)=\operatorname{Ext}_{\mathrm{A}-\mathrm{A}}^{\bullet}(\mathbb{A},-)$ requires such an extension to be unique. So, $H\left(\tilde{\tau}^{\bullet}\right)=\omega^{\bullet}$. Invoking the CCT we obtain the following theorem. 
THEOREM 6.8. If $\mathbb{A}$ is an arbitrary diagram over an arbitrary poset then $H\left(\tilde{\tau}^{\bullet}\right)$ is an isomorphism $H^{\bullet}(\mathbb{A},-) \rightarrow H^{\bullet}(\mathbb{A} !,-!)$.

When $\mathscr{I}$ is finite the CCT can be bypassed in proving that $H\left(\tilde{\tau}^{\bullet}\right)$ is an isomorphism. Indeed, this fact is an immediate consequence of Theorems 6.3 and 6.7. Thus we have an elementary, but less conceptual, proof of a (very) special case of the CCT.

We conclude this section with some remarks on cohomology operations. The Hochschild cohomology of an associative $k$-algebra has an associative gradedcommutative cup product $(\smile)$, a graded Lie bracket $([-,-])$ which acts as graded derivations on the cup product, and a quadratic map (Sq) which is defined on evendimensional cohomology classes. The Lie bracket arises as the graded commutator of the "composition product" ( $\bar{o})$ which, however, is defined only at the cochain level. The quadratic map is given by squaring with respect to the composition product. These products were introduced in $[\mathbf{G 1}]$, although the quadratic map was not named there. (The definitions are repeated in [GS1, 2]. We omit them here.) It follows from the CCT that the Hochschild cohomology of a diagram must have such operations as well. Indeed, given the cochain maps $\tau^{\bullet}$ and $\hat{\tau}^{\bullet}$, the appropriate definitions are clear:

$$
\Gamma \smile \Delta=\hat{\tau}(\tau \Gamma \smile \tau \Delta)
$$

and

$$
\Gamma \bar{\circ} \Delta=\hat{\tau}(\tau \Gamma \bar{o} \tau \Delta)
$$

the Lie bracket and the quadratic map are then defined in the usual way in terms of the composition product. These products can be described directly in terms of $\Gamma$ and $\Delta$. This was done in [GS2] but the formulae were unfortunately misprinted. We repeat them here. We use Steenrod's $\smile_{1}$ which was introduced in $[\mathbf{S}]$ and repeated in [GS2]. If $\Gamma \in C^{m}(\mathbb{A}, \mathbb{M})$ and $\Delta \in C^{n}(\mathbb{A}, \mathbb{A})$ then the formulae for $\Gamma \smile \Delta \in C^{m+n}(\mathbb{A}, \mathbb{M})$ and $\Gamma \bar{o} \Delta \in C^{m+n-1}(\mathbb{A}, \mathbb{M})$ are

$$
\begin{gathered}
(\Gamma \smile \Delta)^{\sigma}=\sum_{\sigma=\nu \smile \nu^{\prime}}(-1)^{\nu^{\prime}(m-\nu)} \Gamma^{\nu} \smile \Delta^{\nu^{\prime}}, \\
(\Gamma \bar{\circ} \Delta)^{\sigma}=\sum_{\sigma=\nu \smile \nu^{\prime}}(-1)^{\nu(n-\nu)} \Gamma^{\nu} \bar{\circ} \Delta^{\nu^{\prime}}-(-1)^{\sigma} \sum_{\sigma= \pm \nu \cup_{1} \nu^{\prime}} \pm \Delta^{\nu^{\prime}} \smile \Gamma^{\nu,}
\end{gathered}
$$

where the \pm is the "sign" of the $\smile_{1}$-factorization $\sigma=\nu \smile_{1} \nu^{\prime}$ (cf. [S, GS2]). These are both graded products. For the first the grading is by dimension; for the second it is by degree (which is one less than dimension). When $\mathbb{M}=\mathbb{A}$ the bracket product of $\Gamma$ and $\Delta$ is their graded commutator $[\Gamma, \Delta] \in C^{n+m-1}(\mathbb{A}, \mathbb{A})$. namely

$$
[\Gamma, \Delta]=\Gamma \bar{\circ} \Delta-(-1)^{(n-1)(m-1)} \Delta \bar{o} \Gamma .
$$

The squaring map $\mathrm{Sq}: C^{2 n}(\mathbb{A}, \mathbb{A}) \rightarrow C^{4 n-1}(\mathbb{A}, \mathbb{A})$ is defined by

$$
\mathrm{Sq}(\Gamma)=\Gamma \overline{ } \bar{\Gamma} .
$$

Cup product, $[-,-]$, and Sq induce products on the cohomology $H^{\bullet}(\mathbb{A}, \mathbb{A})$. (However, $\bar{o}$ does not.) These cohomology operations have all the properties familiar from the classical case. In [GS2] we gave an ad hoc proof of this fact for the case in which the nerve of $\mathscr{I}$ is locally finite. Now that we are armed with the full CCT the fact is trivial and requires no restrictions on $\mathscr{I}$. 
7. Constant diagrams: The product of an algebra with a space. We say that $\mathbb{A}$ is a constant diagram if there is a $k$-algebra $A$ such that $\mathbb{A}^{i}=A$ for all $i \in \mathscr{I}$ and $\varphi^{i j}=\operatorname{Id}_{A}$ for all $i \leq j$, i.e., $\mathbb{A}: \mathscr{J}$ op $\rightarrow k$-alg is a constant functor. Constant bimodules are defined similarly. The most trivial constant diagram, $\mathbb{k}$ is already of interest, for its cohomology is just the simplicial cohomology of $\Sigma$, the nerve of $\mathscr{I}$. We have

THEOREM 7.1. ( $\mathrm{SC}=\mathrm{HC})$. There is an isomorphism $H^{\bullet}(\Sigma, k) \rightarrow H^{\bullet}(\mathbb{k} !, \mathbb{k} !)$ which preserves cup product and Sq.

ProOF. We use the notation of $\S 6$. If $p>0$ then (6.3) reveals that $\bar{C}^{p}(k, k)=0$. Hence, referring to (6.5) we find

$$
\bar{C}^{p}(\mathbf{k}, \mathbf{k})=\prod_{\Sigma_{p}} \bar{C}^{0}(k, k)=\prod_{\Sigma_{p}} k=C^{p}(\Sigma, k),
$$

the usual simplicial $p$-cochain group. Since $\varphi^{i j}=\operatorname{Id}_{k}$ for all $i \leq j$ the coboundary on $\bar{C} \bullet(\mathbf{k}, \mathbb{k})$ reduces to the usual simplicial coboundary and $C^{\bullet}(\Sigma, k)=\bar{C} \bullet(\mathbb{k}, \mathbb{k})$. The CCT now provides the isomorphism. That the isomorphism preserves cohomology operations was proved for a limited class of posets in [GS2, §6]. The proof for an arbitrary poset is identical.

The Lie bracket on $H^{\bullet}(\mathbf{k}$ !, $\mathbf{k}$ !) is abelian [GS2, §6]. The simplices of an arbitrary simplicial complex, when ordered by the incidence relation, form a poset $\mathscr{J}$. The nerve of $\mathscr{I}$ is then the barycentric subdivision of the complex. Thus, the simplicial cohomology (with coefficients in $k$ ) of any simplicial complex equals the Hochschild cohomology of some $k$-algebra. The primitive version of the CCT in [GS1] was strong enough to prove the last mentioned fact for locally finite simplicial complexes, [GS2], but too weak to prove Theorem 7.1. If $\mathscr{I}$ is finite then the CCT is not needed at all to prove $\mathrm{SC}=\mathrm{HC}$. For then, again in the notation of $\S 6$, it is easy to see that $\bar{C} \bullet(\mathbf{k} !, S ; \mathbb{k} !)=C^{\bullet}(\Sigma, k)$ and Theorem 6.3 , which relies only on $(6.1)$, translates to Theorem 7.1. The latter approach to $\mathrm{SC}=\mathrm{HC}$ is the one adopted in [GS4, §2] for finite posets. However, in the case of arbitrary posets (or simplicial complexes) we are unaware of any proof for Theorem 7.1 which avoids the CCT.

The classical concept of a local coefficient system on a simplicial complex coincides with the definition of a $\mathbf{k}$-bimodule $\mathbb{M}$. As in the proof of Theorem 7.1 , it is easy to see that $C^{\bullet}(\Sigma, \mathbb{M})=\bar{C}^{\bullet}(\mathbf{k}, \mathbb{M})$ and, so, $H^{\bullet}(\Sigma, \mathbb{M})=H^{\bullet}(\mathbf{k}$ !, M! $)$. Similarly, $\bar{C} \bullet(\mathbf{k} !, S ; \mathbb{M} !)=C^{\bullet}(\Sigma, \mathbb{M})$ when $\mathscr{I}$ is finite. Thus, classical local cohomologies are also special cases of Hochschild cohomology. In fact, the CCT suggests enlarging the classical concept of a local coefficient system to permit arbitrary $\mathbf{k}$ !-bimodules as coefficients, i.e. set $H^{\bullet}(\Sigma, \mathfrak{b})=H^{\bullet}(\mathbf{k} !, \mathfrak{b})$ for $\mathfrak{b} \in \mathbf{k}$ !-bimod. When $\mathscr{J}$ is finite the cochain complex $\bar{C} \bullet(\mathbf{k}$ !, $S ; \mathfrak{b})$ generalizes the classical cochain complex for local cohomology and Theorem 6.3 informs us that $H^{\bullet}(\Sigma, \mathfrak{b})=H\left(\bar{C}^{\bullet}(\mathbf{k} !, S ; \mathfrak{b})\right)$. What this generalized local cohomology means for simplicial complexes is not yet clear.

As a partial generalization of the finite case of $\mathrm{SC}=\mathrm{HC}$ we have

THEOREM 7.2. Suppose that $k$ is a field, $\mathscr{I}$ is a finite poset, $A$ is a finitedimensional $k$-algebra, and $M$ is an arbitrary $A$-bimodule. Let $\mathbb{A}$ be the constant diagram with $\mathbb{A}^{i}=A$ and let $\mathbb{M}$ be the constant $\mathbb{A}$-bimodule with $\mathbb{M}^{i}=M$. Also let $\Sigma$ be the nerve of $\mathscr{J}$. Then $H^{\bullet}(\mathbb{A} !, \mathbb{M} !) \cong H^{\bullet}(A, M) \otimes H^{\bullet}(\Sigma, k)$. 
Proof. It is trivial that $\mathbb{A} !=A \otimes \mathbb{k} !$ and $\mathbb{M} !=M \otimes \mathbb{k}$ !. Then $[\mathbf{M}, \mathrm{X} .7$, Theorem 4] asserts that $H^{\bullet}(\mathbb{A} !, \mathbb{M} !) \cong H^{\bullet}(A, M) \otimes H^{\bullet}(\mathbb{k}$ !, $\mathbb{k}$ !) and Theorem 7.1 applies to yield the isomorphism.

Note that Theorem 7.2 relies on only the finite case of Theorem 7.1 and that case, in turn, relies on (6.1). Hence Theorem 7.2 does not require the CCT. However, without any finiteness assumptions and over any base ring $k,(6.5)$ easily yields: if $\mathbb{A}$ and $\mathbb{M}$ are constant with $\mathbb{A}^{i}=A$ and $\mathbb{M}^{i}=M$ then $\bar{C} \bullet(\mathbb{A}, \mathbb{M})=\bar{C} \bullet(A, M) \otimes$ $C^{\bullet}(\Sigma, k)$. So, using the CCT, we see that $H^{\bullet}(\mathbb{A} !, \mathbb{M} !)$ is the cohomology of the tensor product of complexes $\bar{C} \bullet(A, M) \otimes C^{\bullet}(\Sigma, k)$.

These theorems and observations provide, at the least, tools for constructing algebras with particular cohomological properties by starting with simpler building blocks. For example, in [GS4] we exploit Theorem 7.1 to construct finitedimensional algebras which are analytically rigid but not infinitesimally rigid. The technique, essentially, is to find a simplicial complex with the right cohomological properties, let $\mathscr{J}$ be the poset formed by its simplices, and then take the resulting $\mathbf{k}$ ! for the algebra.

Finally, let $\mathscr{X}$ be a traingulable space, let $\mathscr{I}$ be the poset formed by the simplices of some triangulation of $\mathscr{X}$, and let $A$ be a $k$-algebra. Then $C^{\bullet}(\mathscr{X}, k)=C^{\bullet}(\Sigma, k)$ and $H^{\bullet}(\mathscr{X}, k)=H^{\bullet}(\Sigma, k)$. Let $\mathbb{A}$ be the constant diagram with $\mathbb{A}^{i}=A$. In view of Theorem 7.2 and the subsequent comments, it seems natural to view $\mathbb{A}$ ! as a "product" of the algebra and the space, $A \times \mathscr{X}$. If $\mathscr{X}$ is compact we may use a finite triangulation. If, in addition, $k$ is a field and $A$ is finite-dimensional then we have, in particular, $H^{\bullet}(A \times \mathscr{X}, A \times \mathscr{X})=H^{\bullet}(A, A) \otimes H^{\bullet}(\mathscr{X}, k)$. For example, consider the poset $\mathscr{J}=\{1,2,3,4\}$ with $1,2<3,4$ (but $1 \nless 2$ and $3 \nless 4$ ). The underlying space of the nerve of $\mathscr{I}$ is the circle, $S^{1}$, and $A \times S^{1}=\mathbb{A}$ ! is the subalgebra of the $4 \times 4$ matrices $M_{4}(A)$ generated by the matrix units $E^{11}, \ldots, E^{44}, E^{13}, E^{14}, E^{23}, E^{24}$. If $k$ is a field and $A$ is finite-dimensional then Theorem 7.2 asserts, for any $A$-bimodule $M$, that $H^{\bullet}\left(A \times S^{1}, M \times S^{1}\right) \cong H^{\bullet}(A, M)+H^{\bullet-1}(A, M)$. In fact, it is not hard to verify the last isomorphism for an arbitrary base ring $k$ and an arbitrary $k$-algebra A.

We believe that such "products" of algebras and spaces are a potential fruitful source of examples and merit further study.

\section{BIBLIOGRAPHY}

[CE] H. Cartan and S. Eilenberg, Homological algebra, Princeton Univ. Press, Princeton, N. J., 1956.

[G1] M. Gerstenhaber, The cohomology structure of an associative ring, Ann. of Math. (2) 78 (1963), 267-288.

[G2] _ On the deformation of rings and algebras, Ann. of Math. (2) 79 (1964), 59-103.

[GS1] M. Gerstenhaber and S. D. Schack, On the deformation of algebra morphisms and diagrams, Trans. Amer. Math. Soc. 279 (1983), 1-50.

[GS2] _ Simplicial cohomology is Hochschild cohomology, J. Pure Appl. Algebra (2) 30 (1983), 143-156.

[GS3] _ On the cohomology of an algebra morphism, J. Algebra (1) 95 (1985), 245-262.

[GS4] _ Relative Hochschild cohomology, rigid algebras, and the Bockstein, J. Pure Appl. Algebra (1) 43 (1986), 53-74.

[GS5] _ The cohomology of presheaves of algebras II: the barycentric subdivision of a small category (to appear).

[GS6] _ , The cohomology of presheaves of algebras III: Embedding theorems (to appear). 
[Gr] A. Grothendieck, Sur quelques points d'algebre homologique, Tôhoku Math. J. 9 (1957), 119221.

[H] G. Hochschild, On the cohomology groups of an associative algebra, Ann. of Math. (2) 46 (1945), 58-67.

[M] S. Mac Lane, Homology, Springer-Verlag, Berlin and New York, 1967.

[S] N. E. Steenrod, Products of cocycles and extensions of mappings, Ann. of Math. (2) 48 (1947), 290-320.

[VZ] O. Villamayor and D. Zelinsky, Galois theory for rings with finitely many idempotents, Nagoya Math. J. 27 (1966), 721-731.

Department of Mathematics, University of Pennsylvania, Philadelphia, PENNSYLVANIA 19104-6395

Department of MAThematics, State University of New York at Buffalo, BUFFALO, NEW YORK 14214-3093 